\title{
THE EVOLUTION OF INTERSTELLAR MEDIUM MASS PROBED BY DUST EMISSION: ALMA OBSERVATIONS AT $z=0.3-2$
}

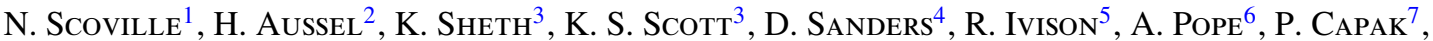 \\ P. Vanden Bout ${ }^{8}$, S. Manohar ${ }^{1}$, J. Kartaltepe ${ }^{9}$, B. Robertson ${ }^{10}$, And S. Lilly ${ }^{11}$ \\ ${ }^{1}$ California Institute of Technology, MC 249-17, 1200 East California Boulevard, Pasadena, CA 91125, USA \\ 2 AIM Unité Mixte de Recherche CEA CNRS, Université Paris VII UMR n158, F-75014 Paris, France \\ ${ }^{3}$ North American ALMA Science Center, National Radio Astronomy Observatory, 520 Edgemont Road, Charlottesville, VA 22901, USA \\ ${ }^{4}$ Institute for Astronomy, 2680 Woodlawn Drive, University of Hawaii, Honolulu, HI 96822, USA \\ ${ }^{5}$ UK Astronomy Technology Centre, Science and Technology Facilities Council, Royal Observatory, Blackford Hill, Edinburgh EH9 3HJ, UK \\ ${ }^{6}$ Department of Astronomy, University of Massachusetts, Amherst, MA 01003, USA \\ ${ }^{7}$ Spitzer Science Center, MS 314-6, California Institute of Technology, Pasadena, CA 91125, USA \\ ${ }^{8}$ National Radio Astronomy Observatory, 520 Edgemont Road, Charlottesville, VA 22901, USA \\ ${ }^{9}$ National Optical Astronomy Observatory, 950 North Cherry Avenue, Tucson, AZ 85719, USA \\ ${ }^{10}$ Department of Astronomy and Steward Observatory, University of Arizona, Tucson, AZ 85721, USA \\ ${ }^{11}$ Institute for Astronomy, ETH Zurich, Wolfgang-Pauli-strasse 27, CH-8093 Zurich, Switzerland \\ Received 2013 September 25; accepted 2014 January 13; published 2014 February 20
}

\begin{abstract}
The use of submillimeter dust continuum emission to probe the mass of interstellar dust and gas in galaxies is empirically calibrated using samples of local star-forming galaxies, Planck observations of the Milky Way, and high-redshift submillimeter galaxies. All of these objects suggest a similar calibration, strongly supporting the view that the Rayleigh-Jeans tail of the dust emission can be used as an accurate and very fast probe of the interstellar medium (ISM) in galaxies. We present ALMA Cycle 0 observations of the Band 7 (350 GHz) dust emission in 107 galaxies from $z=0.2$ to 2.5. Three samples of galaxies with a total of 101 galaxies were stellar-mass-selected from COSMOS to have $M_{*} \simeq 10^{11} M_{\odot}: 37$ at $z \sim 0.4,33$ at $z \sim 0.9$, and 31 at $z=2$. A fourth sample with six infrared-luminous galaxies at $z=2$ was observed for comparison with the purely mass-selected samples. From the fluxes detected in the stacked images for each sample, we find that the ISM content has decreased by a factor $\sim 6$ from 1 to $2 \times 10^{10} M_{\odot}$ at both $z=2$ and 0.9 down to $\sim 2 \times 10^{9} M_{\odot}$ at $z=0.4$. The infrared-luminous sample at $z=$ 2 shows a further $\sim 4$ times increase in $M_{\text {ISM }}$ compared with the equivalent non-infrared-bright sample at the same redshift. The gas mass fractions are $\sim 2 \% \pm 0.5 \%, 12 \% \pm 3 \%, 14 \% \pm 2 \%$, and $53 \% \pm 3 \%$ for the four subsamples $(z=0.4,0.9$, and 2 and infrared-bright galaxies).
\end{abstract}

Key words: dust, extinction - evolution - galaxies: ISM - galaxies: starburst

Online-only material: color figures

\section{INTRODUCTION}

The interstellar medium (ISM) in galaxies is critical to fueling the activities of galactic star formation (SF) and active galactic nuclei (AGNs). Following the peak in cosmic SF at $z=2.5$, the overall star formation rate (SFR) has declined by a factor of 20 over the last 11 Gyr (Hopkins \& Beacom 2006; Carilli \& Walter 2013). Although the ISM fuels the SF, it is unclear if the decline is due to exhaustion of the galactic ISM, less efficient conversion of ISM to stars, or a reduction in galactic accretion of fresh intergalactic medium. At present, there exist inadequate samples of galaxies with measured ISM gas contents to discriminate among these possibilities.

Over the last decade, the rotational transitions of $\mathrm{CO}$ have been used to probe the molecular ISM of high-redshift galaxies. These observations are time consuming and only $\sim 75$ galaxies have been detected and imaged in CO (Baker et al. 2004; Solomon \& Vanden Bout 2005; Coppin et al. 2009; Tacconi et al. 2010, 2013; Bothwell et al. 2013; Carilli \& Walter 2013 and references therein). Most of the existing detections have been for galaxies with ISM masses exceeding $10^{10} M_{\odot}$ or in highly lensed systems. ALMA will improve this situation; still, observing times of $>6 \mathrm{hr}$ will be required for a Milky-Way-like galaxy at $z \sim 2$.

Here, we develop an alternative approach using the long wavelength dust continuum to probe of ISM masses at high redshift (as suggested by Scoville 2012; Eales et al. 2012; Magdis et al. 2013 and references therein). One hundred and seven galaxies in the COSMOS field at $z=0.2$ to 2.5 were observed with ALMA in the continuum at $350 \mathrm{GHz}$ $(\lambda=850 \mu \mathrm{m})$.

In Section 2, we present the physical and empirical basis for using long wavelength dust emission as a probe of ISM mass. The empirical calibration is obtained from (1) a sample of local star-forming galaxies, (2) Planck observations of the Milky Way, and (3) high-redshift submillimeter galaxies (SMGs). All of these yield a similar rest-frame $850 \mu \mathrm{m}$ luminosity per unit ISM mass with a small dispersion. The sample of 107 galaxies for ALMA is presented in Section 3. These galaxies were selected in COSMOS to have relatively constant stellar mass $\left(\sim 10^{11} M_{\odot}\right)$ but span the redshift range $z=0.2-2.5$. Of the 107 galaxies, $26 \%$ were detected individually in integrations ranging from 1 to 4 minutes (with $\sim 15-18$ telescopes in the array and only $5 \mathrm{hr}$ total time). All subsamples were significantly detected in stacked images. The observational results (Section 4 and the Appendix) indicate strong evolution in the ISM contents of these galaxies from $z=2$ to 0.3 .

Our results provide a strong foundation for much larger surveys of dust emission in the future to probe the ISM evolution at high redshift. This approach is much faster $(\sim 30 \times)$ than molecular line observations and more reliably calibrated (given the likely variations in molecular line excitation and 
variations in the $\mathrm{CO}-$ to- $\mathrm{H}_{2}$ conversion factor for higher $\mathrm{CO}$ transitions).

\section{LONG WAVELENGTH DUST CONTINUUM AS A MASS TRACER}

The far-infrared (FIR) to submillimeter emission from galaxies is dominated by dust re-emission of the luminosity from stars and AGNs. The luminosity at the peak of the FIR is often used to estimate the luminosity of obscured SF or AGNs.

Equally important (but not often stressed) is the fact that the long wavelength Rayleigh-Jeans (RJ) tail of dust emission is nearly always optically thin, thus providing a direct probe of the total dust and hence the ISM mass-provided that the dust emissivity per unit mass and the dust-to-gas abundance ratio can be constrained. Fortunately, both of these prerequisites are well established from observations of nearby galaxies (e.g., Draine et al. 2007; Galametz et al. 2011). Theoretical understanding of the dust emissivity has also significantly improved in the last two decades (Draine \& Li 2007).

On the optically thin, RJ tail of the infrared (IR) emission, the observed flux density is given by

$$
S_{v} \propto \kappa_{d}(v) T_{d} v^{2} \frac{M_{d}}{d_{l}^{2}}
$$

where $T_{d}$ is the temperature of the emitting dust grains, $\kappa_{d}(v)$ is the dust opacity per unit mass of dust, $M_{d}$ is the total mass of dust, and $d_{L}$ is the source luminosity distance. (Here, we have assumed the source is at low redshift, thus not including the bandshifting and the compression of the frequency space, which will be added later.)

In nearby normal star-forming galaxies, the majority of the dust is at $T_{d} \sim 20 \rightarrow 35 \mathrm{~K}$ and even in the most vigorous starbursts like Arp 220 the FIR/submillimeter emission is dominated by dust at temperatures $\leqslant 45 \mathrm{~K}$. Thus, variations in the effective dust temperature are small on a galactic scale and the observed fluxes probe the total mass of dust.

Equation (1) can be rewritten in terms of the specific luminosity at the fiducial wavelength $\lambda=850 \mu \mathrm{m}(\nu=$ $353 \mathrm{GHz}$ ). Let $j_{v}$ be the specific emissivity per unit volume (erg s${ }^{-1} \mathrm{~Hz}^{-1}$ ster $^{-1} \mathrm{~cm}^{-3}$ ), $\kappa(v)$ be the dust opacity per unit path length $\left(=n_{d} \sigma_{\nu}\right)$, and vol the volume of the source. The specific luminosity is then

$$
\begin{aligned}
L_{v} & =4 \pi j_{v} \text { vol }=4 \pi \kappa(v) \mathrm{B}_{v}\left(\mathrm{~T}_{\mathrm{d}}\right) \mathrm{vol} \\
& =4 \pi \kappa_{d}(v) \rho_{d} B_{v}\left(T_{d}\right) \mathrm{vol}
\end{aligned}
$$

where $\rho_{d}$ is the dust mass per unit volume in the ISM.

For the RJ tail of the spectrum, this translates to

$$
\alpha_{v} \equiv \frac{L_{v}}{M_{\mathrm{ISM}}}=\frac{8 \pi k}{c^{2}} \kappa_{\mathrm{ISM}}(v) T_{d} \nu^{2},
$$

where $\kappa_{\mathrm{ISM}}(\nu)$ is the dust opacity per $\mathrm{g}$ of ISM mass (i.e., $\left.\kappa_{\mathrm{ISM}}(v)=\kappa_{d}(v) \rho_{d} / \rho_{\mathrm{ISM}}\right)$. Thus,

$$
\begin{aligned}
\alpha_{v} \equiv & \frac{L_{v}}{M_{\mathrm{ISM}}}=\kappa_{\mathrm{ISM}}(v) T_{d}\left(\frac{v}{v_{850 \mu \mathrm{m}}}\right)^{2} \\
& \times 9.54 \times 10^{20} \mathrm{erg} \mathrm{s}^{-1} \mathrm{~Hz}^{-1} M_{\odot}{ }^{-1} .
\end{aligned}
$$

The long wavelength dust opacity can be approximated by a power law in wavelength:

$$
\kappa_{\mathrm{ISM}}(\nu)=\kappa_{\mathrm{ISM}}\left(v_{850 \mu \mathrm{m}}\right)(\lambda / 850 \mu \mathrm{m})^{-\beta} .
$$

The long wavelength opacity per unit mass (Equation (2)) can be investigated using submillimeter observations of nearby galaxies and the Galactic ISM and then applied to the high-redshift galaxies. These local calibrations allow one to ascertain a best estimate for $\kappa_{\mathrm{ISM}}(v)$ and to determine if significant variations arise between atomic and molecular phases of the ISM. There are two aspects to this calibration: (1) the spectral index of $\kappa_{\mathrm{ISM}}(\nu)$ is required to relate flux measures in the different wavelength bands and at different redshifts and (2) $\kappa_{\text {ISM }}\left(v_{850 \mu \mathrm{m}}\right)$, the dust opacity per unit mass at a fiducial wavelength, here chosen to be $\lambda=850 \mu \mathrm{m}\left(v_{850 \mu \mathrm{m}} \equiv 350 \mathrm{GHz}\right)$.

\subsection{The Dust Submillimeter Spectral Index- $\beta$}

The overall spectral slope of the rest-frame submillimeter dust emission flux density (Equation (1)) is observed to vary as $S_{v} \propto v^{\alpha}$ with $\alpha=3-4$. Two powers of $v$ are from the RJ dependence; the remainder is due to the frequency variation in $\kappa_{\text {ISM }}(\nu)\left(\propto v^{\beta}\right)$. Most theoretical models for the dust have opacity spectral indices of $\beta=1.5-2$ (Draine 2011). Empirical fits to the observed long wavelength spectral energy distributions (SEDs) suggest $\beta=1.5$ to 2 (Dunne \& Eales 2001; Clements et al. 2010) for local galaxies. For high- $z$ SMGs, the apparent spectral index is $\alpha=3.2-3.8$, but in some cases the shorter wavelength point is getting close to the IR peak in the rest frame and therefore is not strictly on the RJ tail. Probably the best determination is that of Chapin et al. (2009), who used their $\lambda=1.1$ survey to find $\langle\beta\rangle=1.75$ for 29 SMGs with a median $z=2.7$.

Recently, the Planck mission has provided a robust determination of $\beta$ with 7 bands (at $\lambda=3 \mathrm{~mm}$ to $100 \mu \mathrm{m}$ ) observing the submillimeter dust emission from the Galactic ISM. Planck Collaboration (2011b) analyzed data on the Taurus cloud complex and derived $\beta=1.78 \pm 0.08$, including both atomic and molecular ISM regions. Using the very extensive Planck data throughout the Galaxy, Planck Collaboration (2011a) find $\beta=1.8 \pm 0.1$ with no significant difference between the $\mathrm{H}$ I and $\mathrm{H}_{2}$-dominant regions.

In the remainder of this paper, we adopt $\beta=1.8$, as derived by the very high quality Planck observations of the Galaxy. (This $\beta$ is also consistent with the prior extragalactic determinations, as summarized earlier, within their uncertainties.)

\subsection{Calibration of $\kappa_{I S M}\left(v_{850 \mu m}\right)$ from Local Galaxies and Low-z ULIRGs}

In order to empirically calibrate the dust opacity per unit mass of ISM, we make use of galaxy samples for which both the submillimeter dust emission and ISM masses are well determined globally (or where the measurements refer to the same areas in the galaxy).

Our local galaxy sample includes SCUBA data for 12 galaxies-3 from the SINGS survey (Dale et al. 2005) and 9 from the ultraluminous infrared galaxies (ULIRGs) survey (Clements et al. 2010). The ULIRG sample is in fact probably most relevant since these galaxies are closer in IR luminosity to the high-redshift galaxies and very importantly, their emission is compact so we can be confident that the total $850 \mu \mathrm{m}$ flux and ISM masses encompass the same regions. (As a reliability check for the submillimeter measurements, we required observed fluxes at both 450 and $850 \mu \mathrm{m}$ and that their ratios yield a reasonable spectral index.) The submillimeter fluxes and ISM masses for the low $-z$ galaxy sample are provided in Table 1. 
Table 1

Low- $z$ Galaxies with Submillimeter and ISM Data

\begin{tabular}{lrcccr}
\hline \hline Galaxy & $\begin{array}{c}\text { Distance } \\
(\mathrm{Mpc})\end{array}$ & $\begin{array}{c}S_{\nu}(450 \mu \mathrm{m}) \\
(\mathrm{Jy})\end{array}$ & $\begin{array}{c}S_{\nu}(850 \mu \mathrm{m}) \\
(\mathrm{Jy})\end{array}$ & $\begin{array}{c}\log M_{\mathrm{H}_{\mathrm{I}}} \\
\left(M_{\odot}\right)\end{array}$ & $\begin{array}{c}\log M_{\mathrm{H}_{2}} \\
\left(M_{\odot}\right)\end{array}$ \\
\hline NGC 4631 & 9.0 & 30.7 & 5.73 & 9.2 & 9.5 \\
NGC 7331 & 15.7 & 18.5 & 2.98 & 9.4 & 9.7 \\
NGC 7552 & 22.3 & 20.6 & 2.11 & 9.7 & 10.0 \\
NGC 598 & 76.0 & 2.3 & 0.26 & 9.8 & 10.1 \\
NGC 1614 & 62.0 & 1.0 & 0.22 & 9.7 & 10.0 \\
NGC 1667 & 59.0 & 1.2 & 0.16 & 9.3 & 9.6 \\
Arp 148 & 143.0 & 0.6 & 0.09 & 9.9 & 10.2 \\
1ZW107 & 170.0 & 0.4 & 0.06 & 10.0 & 10.3 \\
Arp 220 & 79.0 & 6.3 & 0.46 & 10.0 & 10.3 \\
12112+0305 & 293.0 & 0.5 & 0.05 & 10.3 & 10.6 \\
Mrk 231 & 174.0 & 0.5 & 0.08 & 9.8 & 10.1 \\
Mrk 273 & 153.0 & 0.7 & 0.08 & 9.9 & 10.2 \\
\hline
\end{tabular}

Notes. Submillimeter flux measurements and ISM masses from Dale et al. (2005), Clements et al. (2010), and the NASA Extragalactic Database. Hi masses were set to $50 \%$ of the molecular gas masses. Uncertainties in the derived $\mathrm{H}_{2}$ masses are dominated by the assumed CO-to- $\mathrm{H}_{2}$ conversion factor, which is uncertain by $\sim \pm 25 \%$ even for normal low-level star-forming galaxies.

The $850 \mu \mathrm{m}$ fluxes were converted to specific luminosity $L_{v(850 \mu \mathrm{m})}$, using

$$
\begin{aligned}
L_{v} & =10^{-23} 4 \pi d_{L}^{2} S_{\nu}(\mathrm{Jy}) \\
& =1.19 \times 10^{27} S_{v}(\mathrm{Jy}) d_{L}^{2}(\mathrm{Mpc}) \mathrm{erg} \mathrm{s}^{-1} \mathrm{~Hz}^{-1}
\end{aligned}
$$

and ratioed to the ISM masses $\left(\mathrm{HI}\right.$ and $\mathrm{H}_{2}$, Figure 1). Based on the data shown in Figure 1, we find a constant of proportionality between the $850 \mu \mathrm{m}$ specific luminosity and the ISM mass:

$$
\begin{aligned}
& \alpha_{850 \mu \mathrm{m}}=\frac{L_{\nu_{850 \mu \mathrm{m}}}}{M_{\mathrm{ISM}}} \\
&=1.0 \pm 0.23 \times 10^{20} \mathrm{erg} \mathrm{s}^{-1} \mathrm{~Hz}^{-1} M_{\odot}{ }^{-1} . \\
& \text { 2.3. } \kappa_{I S M}(850 \mu \mathrm{m}) \text { in the Galaxy from Planck }
\end{aligned}
$$

The Planck measurements of the submillimeter emission from the Galaxy provide both very high photometric accuracy and the ability to probe variations in the opacity-to-mass ratio between atomic and molecular phases and with Galactic radius. The latter provides a weak probe of metallicity dependence.

In the Taurus complex, Planck Collaboration (2011b) obtained best-fit ratios of $\tau_{250 \mu \mathrm{m}} / N_{\mathrm{H}}=1.1 \pm 0.2$ and $2.32 \pm 0.3 \times$ $10^{-25} \mathrm{~cm}^{2}$ for the atomic and molecular ${ }^{12}$ phases, respectively. (The former is consistent with a much earlier determination of $1 \times 10^{25} \mathrm{~cm}^{2}$ (Boulanger et al. 1996) for the diffuse ISM.)

Using Planck data from the Galaxy, Planck Collaboration (2011a) found $\tau_{250 \mu \mathrm{m}} / N_{\mathrm{H}}=0.92 \pm 0.05 \times 10^{-25} \mathrm{~cm}^{2}$ near the solar circle with no significant variation in Galactic radius. This includes the inner galaxy-where the molecular phase dominates and the mean dust temperatures are higher-in addition to the solar circle and outer radii where $\mathrm{H}$ I dominates. It would appear that there is no strong evidence of variation in the opacity per unit mass with either ISM phase, dust temperature, or

\footnotetext{
12 We note that the estimation of the molecular gas masses by Planck Collaboration (2011a) assumed a value $X_{\mathrm{CO}}=2 \times 10^{20} \mathrm{H}_{2} \mathrm{~cm}^{-2}$ $\left(\mathrm{K} \mathrm{km} \mathrm{s}^{-1}\right)^{-1}$ based on Fermi gamma ray observations. However, virial mass estimates for the giant molecular clouds indicate a best

$X_{\mathrm{CO}}=3.6 \pm 0.2 \times 10^{20}$ (Scoville et al. 1987), implying higher $\mathrm{H}_{2}$ masses and therefore a value $\tau_{250 \mu \mathrm{m}} / N_{\mathrm{H}}=1.3 \times 10^{-25} \mathrm{~cm}^{2}$, i.e., more similar to the atomic phase value.
}

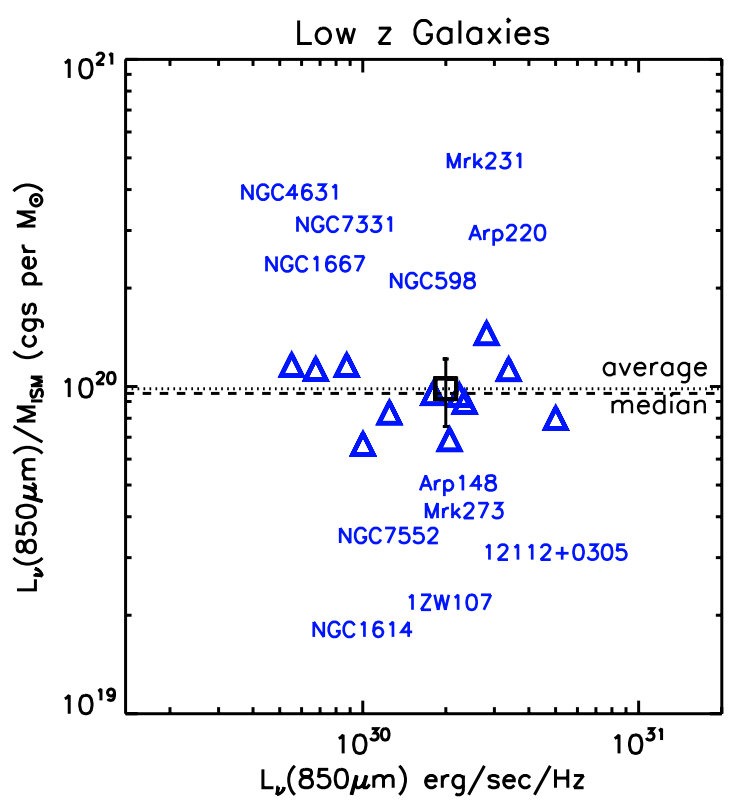

Figure 1. Ratio of $L_{v}$ at $850 \mu \mathrm{m}$ to $M_{\mathrm{ISM}}$ is shown for a sample of low- $z$ spiral and starburst galaxies from Dale et al. (2005) and Clements et al. (2010). The average and median values for the sample are shown by horizontal lines. The mean ratio shown by the error bars is $1.00 \pm 0.23 \times 10^{20} \mathrm{erg} \mathrm{s}^{-1} \mathrm{~Hz}^{-1} M_{\odot}{ }^{-1}$. Typical uncertainties in the derived ISM masses are $\pm 50 \%$ due to variation in the CO-to- $\mathrm{H}_{2}$ conversion factor.

(A color version of this figure is available in the online journal.)

metallicity. (The overall range of metallicity probed in the Milky Way is not large so this issue would need to be reexamined using data from low-metallicity dwarf galaxies.)

In the following, we adopt $\tau_{250 \mu \mathrm{m}} / N_{\mathrm{H}} \simeq 1.0 \times 10^{-25} \mathrm{~cm}^{2}$. To convert the scale factors from the Planck analysis to $\kappa_{\mathrm{ISM}}\left(\nu_{850 \mu \mathrm{m}}\right)$, we note that $N_{\mathrm{H}}=\Sigma_{\mathrm{ISM}} /\left(1.36 \mathrm{~m}_{\mathrm{H}}\right)$, where $\Sigma_{\mathrm{ISM}}$ is the total mass column density of ISM and the factor 1.36 is to account for $\mathrm{He}$ at $9 \%$ abundance. Using $\beta=1.8$ to scale from 250 to $850 \mu \mathrm{m}$ implies an additional factor 0.11 and thus

$\kappa_{\mathrm{ISM}}\left(v_{850 \mu \mathrm{m}}\right)=\frac{\tau_{250 \mu \mathrm{m}}}{N_{\mathrm{H}}} \frac{0.11}{1.36 \mathrm{~m}_{\mathrm{H}}}=4.84 \times 10^{-3} \mathrm{~g}^{-1} \mathrm{~cm}^{2}$.

The specific luminosity emitted per unit mass of ISM is then given by

$$
\begin{aligned}
\alpha_{850 \mu \mathrm{m}} & =\frac{L_{\nu_{850 \mu \mathrm{m}}}}{M_{\mathrm{ISM}}}=4 \pi \kappa_{\mathrm{ISM}}\left(v_{850 \mu \mathrm{m}}\right) B_{v}\left(T_{d}\right) \\
& =0.79 \times 10^{20} \mathrm{erg} \mathrm{s}^{-1} \mathrm{~Hz}^{-1} M_{\odot}^{-1},
\end{aligned}
$$

where the Planck function was evaluated with $T_{d}=25 \mathrm{~K}$, as discussed immediately below (Section 2.4). (Equation (9) uses the actual Planck function, not the RJ approximation.)

The value of the constant of proportionality between the $850 \mu \mathrm{m}$ flux and the ISM mass in the Galaxy found by the Planck mission is therefore within $20 \%$ of that found earlier in the sample of nearby galaxies.

\subsection{Redshifted Submillimeter Fluxes}

For high redshift, one may estimate the expected submillimeter continuum fluxes using the calibration of specific luminosity at $850 \mu \mathrm{m}$ to ISM mass from Equation (7) and a power law with $\beta=1.8$ for the long wavelength dust opacity. The observed flux 


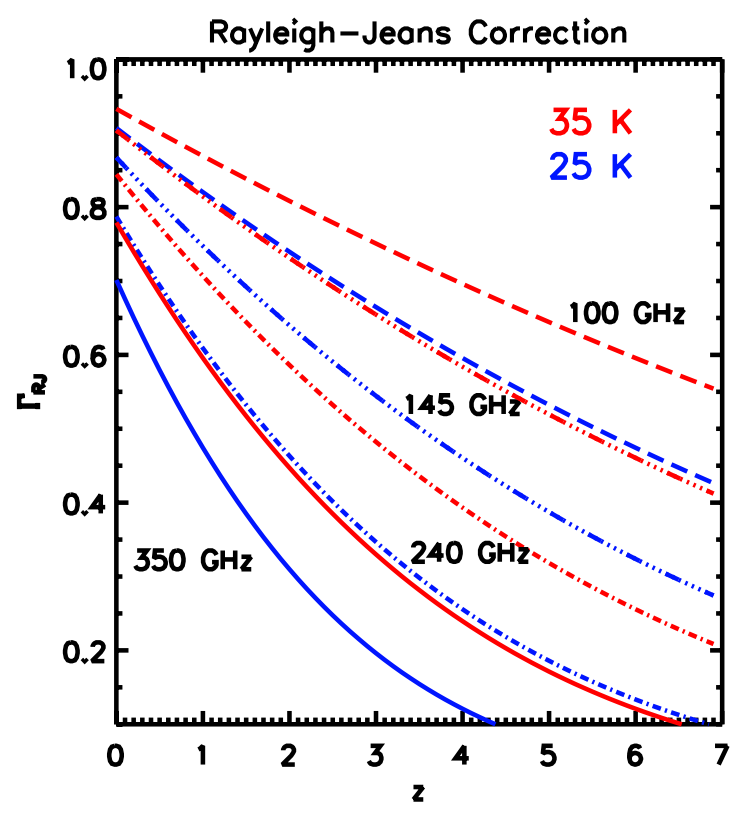

Figure 2. RJ correction factor $\Gamma_{\mathrm{RJ}}$ is shown as a function of redshift for four observing frequencies and dust temperatures of 25 and $35 \mathrm{~K}$.

(A color version of this figure is available in the online journal.)

density is

$$
S_{v_{\mathrm{obs}}}=\alpha_{850 \mu \mathrm{m}} M_{\mathrm{ISM}}(1+z)^{4.8}\left(\frac{v_{\mathrm{obs}}}{v_{850 \mu \mathrm{m}}}\right)^{3.8} \frac{\Gamma_{\mathrm{RJ}}}{\Gamma_{0}} \frac{1}{4 \pi d_{L}^{2}},
$$

where the additional factor of $(1+z)$ beyond that associated with the rest-frame SED is due to the compression of observed frequency space relative to that in the rest frame.

$\Gamma_{\mathrm{RJ}}$ is the correction factor for departures from the RJ $v^{2}$ dependence as the observed emission approaches the SED peak in the rest frame. This can be a substantial correction; it is given by

$$
\Gamma_{\mathrm{RJ}}\left(T_{d}, v_{\mathrm{obs}}, z\right)=\frac{h v_{\mathrm{obs}}(1+z) / k T_{d}}{e^{h v_{\mathrm{obs}}(1+z) / k T_{d}}-1}
$$

and shown in Figure 2 for dust temperatures of 25 and $35 \mathrm{~K}$. ( $\Gamma_{0}$ is the value of $\Gamma$ appropriate to the low $-z \lambda=850 \mu \mathrm{m}$ used to calibrate $\alpha_{850 \mu \mathrm{m}}$; nominally $\Gamma_{0}=0.71$; see Figure 2.)

In the following, we assume $T_{d}=25 \mathrm{~K}$ to characterize the bulk of the ISM mass. (In the Planck observations of the Milky Way, the median was $T_{d} \simeq 18 \mathrm{~K}$ (Planck Collaboration 2011a) but more active galaxies are likely to have somewhat higher dust temperatures.) Dunne et al. (2011) derived dust temperatures in the range 17 to $30 \mathrm{~K}$ for 1867 galaxies at $z<0.5$ in the HerschelATLAS survey. Very similar distributions of dust temperatures were found by Dale et al. (2012) for 61 galaxies in the Herschel KINGFISH survey and Auld et al. (2013) for 254 galaxies in the Herschel Virgo Cluster Survey.

For a flux density measurement at observed frequency $v_{\text {obs }}$,

$$
\begin{aligned}
& S_{v_{\mathrm{obs}}}= 0.83 \frac{M_{\mathrm{ISM}}}{10^{10} M_{\odot}}(1+z)^{4.8}\left(\frac{v_{\mathrm{obs}}}{v_{850 \mu \mathrm{m}}}\right)^{3.8} \\
& \times \frac{\Gamma_{\mathrm{RJ}}}{\Gamma_{0}}\left(\frac{\mathrm{Gpc}}{d_{L}}\right)^{2} \mathrm{mJy} \\
& \text { for } \quad \lambda_{\text {rest }} \gtrsim 250 \mu \mathrm{m},
\end{aligned}
$$

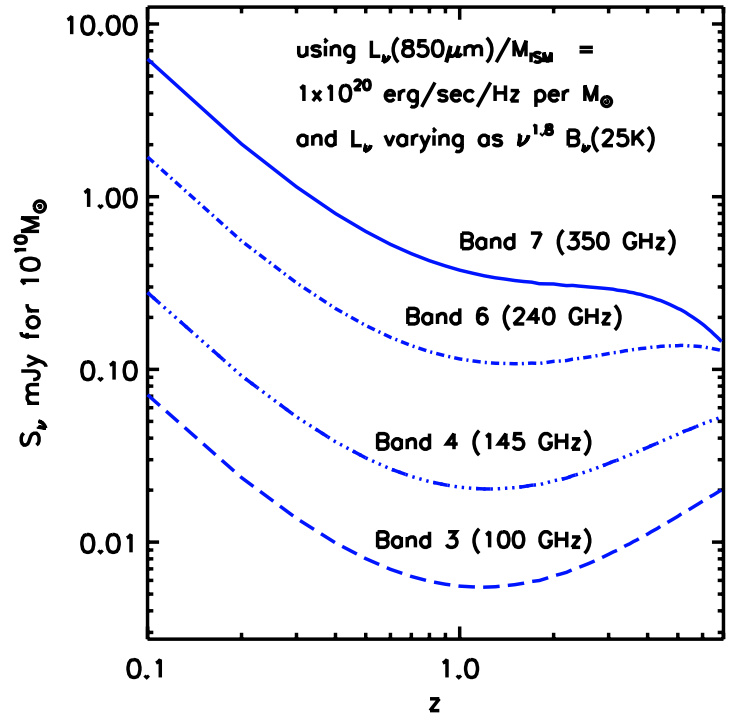

Figure 3. Expected continuum fluxes at $100,145,240$, and $350 \mathrm{GHz}$ for $M_{\text {ISM }}=$ $10^{10} M_{\odot}$ derived using the empirical calibration embodied in Equation (7), an emissivity power-law index $\beta=1.8$, and including the RJ departure coefficient $\Gamma_{\mathrm{RJ}}(25 \mathrm{~K})$. Since the point source sensitivities of ALMA in the three bands are fairly similar, it is clear that the optimum strategy is to use Band 7 out to $z \sim 2$, before moving to lower frequency bands to avoid the RJ correction issues.

(A color version of this figure is available in the online journal.)

where we have used $\alpha_{850 \mu \mathrm{m}}$ given by Equation (7). We note that the empirical calibration of $\alpha_{850}$ was obtained from $z \simeq 0$ galaxies; however, there is still a non-negligible RJ departure $(\sim 0.7)$ that must be normalized out (i.e., the $y$-axis intercept in Figure 2). This is the term $\Gamma_{0}=\Gamma_{\mathrm{RJ}}\left(0, T_{d}, v_{850}\right)$ in the equation above.

The restriction $\lambda_{\text {rest }} \gtrsim 250 \mu \mathrm{m}$ is intended to ensure that one is on the RJ tail and that the dust is likely to be optically thin. If the dust is extremely cold, one might need to be more restrictive and in the case of the most extreme ULIRGs the dust is probably optically thick to even longer wavelengths. Analogous expressions can easily be obtained for the other ALMA bands.

Figure 3 shows the expected flux as a function of redshift for the ALMA bands at 100, 145, 240, and $350 \mathrm{GHz}$ (Bands 3, 4,6 , and 7). At low $z$, the increasing luminosity distance leads to reduced flux as $z$ increases. However, above $z=1$ the wellknown "negative $k$-correction" causes the flux per unit ISM mass to increase at higher $z$ as one moves up the FIR SED toward the peak at $\lambda \sim 100 \mu \mathrm{m}$. Figure 3 shows that the $350 \mathrm{GHz}$ flux density plateaus and then decreases above $z=2$; this is due to the fact that at higher redshift, $350 \mathrm{GHz}$ is approaching the rest-frame FIR peak (and no longer on the $v^{2} \mathrm{RJ}$ tail). This is the factor $\Gamma_{\mathrm{RJ}}$ coming in for $25 \mathrm{~K}$ dust.

At redshifts above 2.5, Figure 3 indicates that one needs to shift to a lower frequency band, i.e., 240, 145, or $100 \mathrm{GHz}$, in order to avoid the large and uncertain $\Gamma_{\mathrm{RJ}}$ corrections. Since future studies similar to that pursued here will push to higher redshifts, we have included the lower frequency bands in Figures 2 and 3.

\subsection{Comparison with High-z SMGs}

The empirical value for $\alpha_{850}\left(=L_{\nu_{850 \mu \mathrm{m}}} / M_{\text {ISM }}\right)$ obtained in Sections 2.2 and 2.3 based on local galaxies and Planck observations of the Milky Way pertain to low-redshift galaxies. We now compare these local calibrations with observations for a large sample of SMGs from the literature (see Table 2). 
Table 2

SMGs with $\mathrm{CO}(1-0)$ Data

\begin{tabular}{|c|c|c|c|c|c|c|c|c|c|c|}
\hline SMG & Ref. & $\mathrm{z}$ & $\begin{array}{c}S_{\nu}(850 \mu) \\
\mathrm{mJy}\end{array}$ & $\mathrm{S} / \mathrm{N}_{850 \mu \mathrm{m}^{\mathrm{a}}}$ & $\frac{\Gamma_{0}}{\Gamma_{\mathrm{RJ}}}$ & $\begin{array}{c}I_{\mathrm{CO}} \\
\mathrm{Jy} \mathrm{km} \mathrm{s}^{-1}\end{array}$ & $\mathrm{~S} / \mathrm{N}_{\mathrm{CO}}{ }^{\mathrm{a}}$ & $\begin{array}{c}M_{\mathrm{ISM}}{ }^{\mathrm{b}} \\
\left(10^{11} M_{\odot}\right)\end{array}$ & $\begin{array}{c}L_{850} \\
\left(10^{31} \mathrm{cgs}\right)\end{array}$ & $\begin{array}{c}L_{850} / M_{\mathrm{ISM}^{\mathrm{b}}} \\
\left(10^{20} \operatorname{cgs} M_{\odot}{ }^{-1}\right)\end{array}$ \\
\hline НXMM01... & 1 & 2.31 & 27.0 & 9.0 & 2.6 & 1.73 & 5.6 & $20.1 \pm 3.6$ & 10.5 & $0.5 \pm 0.2$ \\
\hline SPT-S053816-... & 2 & 2.79 & 125.0 & 17.9 & 3.2 & 1.20 & 6.0 & $19.3 \pm 3.2$ & 47.9 & $2.5 \pm 0.6$ \\
\hline HATLASJ08493... & 3 & 2.41 & 19.0 & 9.5 & 2.7 & 0.56 & 8.0 & $7.0 \pm 0.9$ & 7.1 & $1.0 \pm 0.2$ \\
\hline H-ATLASJ0903... & 4 & 2.31 & 54.7 & 17.6 & 2.6 & 1.00 & 7.7 & $11.6 \pm 1.5$ & 21.2 & $1.8 \pm 0.3$ \\
\hline H-ATLASJ0913... & 4 & 2.63 & 36.7 & 9.4 & 3.0 & 0.76 & 6.3 & $11.1 \pm 1.7$ & 14.6 & $1.3 \pm 0.3$ \\
\hline H-ATLASJ0918... & 4 & 2.58 & 18.8 & 11.8 & 2.9 & 1.04 & 4.0 & $14.7 \pm 3.7$ & 7.4 & $0.5 \pm 0.2$ \\
\hline HLSW-01... & 5 & 2.96 & 52.8 & 105.6 & 3.5 & 1.14 & 10.4 & $20.2 \pm 2.0$ & 21.4 & $1.1 \pm 0.1$ \\
\hline H-ATLASJ1132... & 4 & 2.58 & 106.0 & 5.9 & 2.9 & 0.66 & 3.5 & $9.3 \pm 2.7$ & 4.9 & $0.5 \pm 0.2$ \\
\hline H-ATLASJ1158... & 4 & 2.19 & 107.0 & 5.9 & 2.5 & 0.74 & 6.2 & $7.9 \pm 1.3$ & 4.9 & $0.6 \pm 0.2$ \\
\hline H-ATLASJ1336... & 4 & 2.20 & 36.8 & 12.7 & 2.5 & 0.93 & 7.8 & $10.0 \pm 1.3$ & 14.3 & $1.4 \pm 0.3$ \\
\hline H-ATLASJ1344... & 4 & 2.30 & 73.1 & 30.5 & 2.6 & 2.74 & 7.0 & $31.7 \pm 4.5$ & 28.4 & $0.9 \pm 0.2$ \\
\hline H-ATLASJ1413... & 4 & 2.48 & 33.3 & 12.8 & 2.8 & 1.47 & 8.6 & $19.4 \pm 2.2$ & 13.1 & $0.7 \pm 0.1$ \\
\hline SMMJ2135-010... & 6 & 2.33 & 106.0 & 8.8 & 2.6 & 2.25 & 9.8 & $26.5 \pm 2.7$ & 39.4 & $1.5 \pm 0.3$ \\
\hline SPT-S233227-... & 2 & 2.73 & 150.0 & 13.6 & 3.1 & 1.70 & 6.8 & $26.4 \pm 3.9$ & 57.3 & $2.2 \pm 0.5$ \\
\hline SMMJ123549.4... & 7 & 2.20 & 8.3 & 3.3 & 2.5 & 0.32 & 8.0 & $3.4 \pm 0.4$ & 2.8 & $0.8 \pm 0.3$ \\
\hline SMMJ123707.2... & 7 & 2.49 & 10.7 & 4.0 & 2.8 & 0.91 & 7.0 & $12.1 \pm 1.7$ & 3.7 & $0.3 \pm 0.1$ \\
\hline SMMJ163650.4... & 7 & 2.38 & 8.2 & 4.8 & 2.7 & 0.34 & 8.5 & $4.2 \pm 0.5$ & 2.8 & $0.7 \pm 0.2$ \\
\hline SMMJ163658.1... & 7 & 2.45 & 10.7 & 5.3 & 2.8 & 0.37 & 5.3 & $4.8 \pm 0.9$ & 3.7 & $0.8 \pm 0.3$ \\
\hline EROJ164502+4... & 9 & 1.44 & 4.9 & 6.6 & 1.8 & 0.60 & 6.0 & $3.0 \pm 0.5$ & 1.5 & $0.5 \pm 0.2$ \\
\hline SMMJ02399-01... & 10 & 2.81 & 23.0 & 12.1 & 3.3 & 0.60 & 5.0 & $9.8 \pm 2.0$ & 8.1 & $0.8 \pm 0.2$ \\
\hline SMMJ04135+10... & 10 & 2.85 & 25.0 & 8.9 & 3.3 & 0.64 & 7.9 & $10.7 \pm 1.4$ & 8.8 & $0.8 \pm 0.2$ \\
\hline SMMJ04431+02... & 11 & 2.51 & 7.2 & 4.8 & 2.8 & 0.26 & 4.3 & $3.5 \pm 0.8$ & 2.5 & $0.7 \pm 0.3$ \\
\hline SMMJ14009+02... & 10 & 2.93 & 15.6 & 8.2 & 3.5 & 0.31 & 15.5 & $5.4 \pm 0.3$ & 5.5 & $1.0 \pm 0.2$ \\
\hline SMMJ14011+02... & 10 & 2.57 & 12.3 & 7.2 & 2.9 & 0.40 & 8.0 & $5.6 \pm 0.7$ & 4.3 & $0.8 \pm 0.2$ \\
\hline SMMJ163555.2... & 10 & 2.52 & 12.5 & 15.6 & 2.9 & 0.22 & 5.5 & $3.0 \pm 0.5$ & 4.3 & $1.4 \pm 0.4$ \\
\hline SMMJ163554.2... & 12 & 2.52 & 15.9 & 22.7 & 2.9 & 0.40 & 10.0 & $5.4 \pm 0.5$ & 5.5 & $1.0 \pm 0.1$ \\
\hline SMMJ163550.9... & 12 & 2.52 & 8.4 & 10.5 & 2.9 & 0.30 & 3.3 & $4.1 \pm 1.2$ & 2.9 & $0.7 \pm 0.3$ \\
\hline HATLASJ08493... & 12 & 2.41 & 25.0 & 12.5 & 2.7 & 0.49 & 8.2 & $\begin{array}{r}6.1 \pm 0.8 \\
\text { Average }^{c}:\end{array}$ & $\begin{array}{c}9.4 \\
1.01 \pm 0.52\end{array}$ & $1.5 \pm 0.3$ \\
\hline
\end{tabular}

Notes. Submillimeter fluxes and CO(1-0) measurements from references given in the second column: 1: Fu et al. (2013), 2: Aravena et al. (2013), 3: Ivison et al. (2013), 4: Harris et al. (2012), 5: Riechers et al. (2011b), 6: Lestrade et al. (2011), 7: Ivison et al. (2011), 8: Riechers et al. (2011a), 9: Greve et al. (2003), 10: Thomson et al. (2012), 11: Harris et al. (2010), 12: Ivison et al. (2013), Bussmann et al. (2013).

${ }^{a} \mathrm{~S} / \mathrm{N}$ calculated from the ratio of the measured flux to the stated uncertainty in the observational reference.

${ }^{b}$ Specified uncertainties in the ISM masses and the luminosity-to-mass ratios include only the flux uncertainties for the CO and $850 \mu \mathrm{m}$ measurements. The uncertainty due to variations in the CO-to- $\mathrm{H}_{2}$ conversion ratio is difficult to quantify since the physical conditions in the galaxies are unresolved. It is worth noting that for a large sample of Galactic GMCs with both CO (1-0) luminosities and virial masses, Scoville et al. (1987) measured a dispersion of only 7\% in the ratio $L_{\mathrm{CO}} / M_{\mathrm{vir}}$ and this sample included clouds with and without $\mathrm{H}$ II regions and covered a mass range $1-16 \times 10^{5} M_{\odot}$.

${ }^{c}$ Unweighted average and standard deviation.

We restrict this comparison to SMGs at $z<3$ so that the observed $850 \mu \mathrm{m}$ flux measures will be longward of the FIR peak in the rest frame.

We also restrict our sample to only those SMGs with high signal-to-noise ratio $(\mathrm{S} / \mathrm{N})$ measurements of $\mathrm{CO}(1-0)$. From these, we can reliably obtain ISM mass estimates without large excitation corrections and uncertainties. Although many galaxies have detectable emission in higher $\mathrm{CO}$ transitions, this high excitation gas very likely constitutes just a small fraction of the total molecular gas. The bulk of the molecular gas is probably at $10-20 \mathrm{~K}$ and therefore emits appreciably only in the first couple of $\mathrm{CO}$ lines. Furthermore, the $\mathrm{CO}(1-0)$ line luminosity is well known to be linearly correlated with the gravitational masses of giant molecular clouds (GMCs; Scoville et al. 1987; Solomon et al. 1987). Although some of these SMGs are likely to be lensed, we do not expect a differential magnification difference between the cold molecular gas and the cold dust emission; hence, their ratios should still provide a check on the high-redshift luminosity-to-mass ratio.

The molecular ISM masses were derived from the observed $\mathrm{CO}(1-0)$ line fluxes using Equation (3) in Solomon
$\&$ Vanden Bout (2005) and a standard $\alpha_{\mathrm{CO}}=M_{\mathrm{ISM}} / L_{\mathrm{CO}}=$ 4.6 $M_{\odot}\left(\mathrm{K} \mathrm{km} \mathrm{s}^{-1} \mathrm{pc}^{2}\right)^{-1}$. Columns 9 and 10 list the derived ISM masses and $L_{v_{850 \mu \mathrm{m}}}$. The individual galaxy ratios are given in the last column and are shown in Figure 4 along with the sample average:

$$
\left\langle\alpha_{850 \mu \mathrm{m}}\right\rangle_{\mathrm{SMG}}=1.0 \pm 0.5 \times 10^{20} \mathrm{erg} \mathrm{s}^{-1} \mathrm{~Hz}^{-1} M_{\odot}{ }^{-1} .
$$

In the above analysis, we used the standard Galactic factor $\alpha_{\mathrm{CO}}$ to convert observed $\mathrm{CO}(1-0)$ luminosity to gas mass. As discussed in Solomon \& Vanden Bout (2005), low- $z$ studies of ULIRGs have led to the suggestion that the conversion factor could be several times smaller (Downes et al. 1993). In the ULIRGs, the gas is concentrated in the nuclear regions as a result of dissipative galaxy merging and the molecular gas line width can be increased by the galactic dynamics, including the stellar mass-not just the gas mass, as in the self-gravitating GMCs where the standard conversion factor was derived. In addition, the mean gas temperature and density $(\rho)$ may be different in the ULIRG nuclei as a result of the intense SF activity and $\alpha_{\mathrm{CO}}$ 


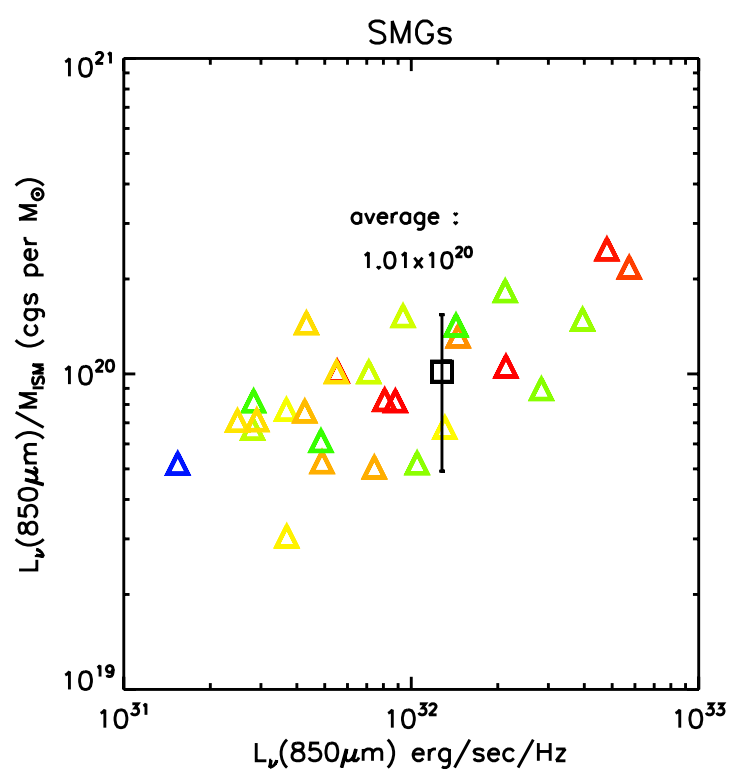

Figure 4. Ratio of $L_{v}$ at $850 \mu \mathrm{m}$ to $M_{\mathrm{ISM}}$ is shown for a sample of $z<3$ SMGs with $\mathrm{CO}(1-0)$ data (references given in Table 2). Colors of the symbols indicate the redshifts ranging from $z=1.44$ (blue) to 3.0 (red). The average value of the luminosity-to-mass ratio $=1.01 \pm 0.52 \times 10^{20} \mathrm{erg} \mathrm{cm}^{-2} \mathrm{~s}^{-1} M_{\odot}{ }^{-1}$ is indicated by the square. (The median value is $8 \times 10^{19} \mathrm{erg} \mathrm{cm}^{-2} \mathrm{~s}^{-1} M_{\odot}{ }^{-1}$.)

(A color version of this figure is available in the online journal.)

should vary as $\langle\rho\rangle^{1 / 2} / T_{k}$ (see Equation (8.5) in Scoville 2012).

For the high- $z$ SMGs, it is not obvious that the lower $\alpha_{\mathrm{CO}}$ (used in low $z$ ULIRGs) is appropriate since it is uncertain that the bulk of the molecular gas in the SMGs is similarly concentrated. Our restriction to $\mathrm{CO}(1-0)$ in the above sample was specifically intended to avoid sensitivity to the presence of high excitation gas and to sample the larger, presumably extended masses of cold gas. Indeed, the ratio of dust emission to gas mass is similar to that obtained in low $-z$ galaxies; this suggests that the SMG $\mathrm{CO}(1-0)$ emission is not enhanced by concentration in the galactic nuclei.

\subsection{Summary: An Approximately Constant RJ Mass-to-Light Ratio}

In the preceding sections, we have presented a physical explanation and, more importantly, strong empirical justification for using the long wavelength RJ dust emission in galaxies as a linear probe of the ISM mass. The most substantial determination of the dust RJ spectral slope is that obtained by the Planck mission from observations of the Galaxy (Planck Collaboration 2011a, 2011b), indicating a dust emissivity index $\beta=1.8 \pm 0.1$ with no strong evidence for variation in Galactic radius or between atomic and molecular regions. Second, both the Planck data and measurements for nearby local galaxies, including both normal star-forming and star-bursting systems, indicate a similar constant of proportionality $\alpha_{850}$ for the dust emission at rest-frame $850 \mu \mathrm{m}$ per unit mass of ISM. Last, we find for a large sample of SMGs at $z=1.4$ to 3 that their ratio of rest-frame $850 \mu \mathrm{m}$ per unit mass of ISM is essentially identical to that obtained for the local galaxies. The similarities of the $\alpha_{850} \mathrm{~s}$ argue strongly that for the majority of ISM mass, the dust emissivity at long wavelengths, the dustto-gas mass ratio, and the dust temperatures vary little. The dispersions (or uncertainties) in the derived $\alpha_{850 \mu m}$ and $\beta$ are $5 \%-25 \%$. Since these uncertainties are generally less than the flux measurement fractional uncertainties, we include only the latter in the following uncertainty estimates.

The submillimeter flux-to-dust mass ratio is expected to vary linearly with dust temperature. In practice, the overall range of $T_{d}$ for the bulk of the mass of ISM is very small, since it requires very large increases in the radiative heating to increase the dust temperatures $\left(T_{d}\right.$ varies approximately as the $1 / 5-1 / 6$ power of the radiation energy density). As noted above, the extensive surveys of local galaxies with Herschel find a range of $T_{d} \sim 15-30 \mathrm{~K}$ (Dunne et al. 2011; Dale et al. 2012; Auld et al. 2013). Where we have needed to specify a dust temperature (e.g., for the RJ correction), we used $25 \mathrm{~K}$, so we expect the uncertainties in the derived masses averaged on galaxy scales will be less than $\sim 25 \%$.

These calibrations, including atomic- and moleculardominant regions, normal to star-bursting systems, inner to outer galaxy, and low to moderate redshift, lay a solid foundation for using measurements of the RJ dust emission to probe galactic ISM masses. ALMA enables this technique for high-redshift surveys, providing high sensitivity and the requisite angular resolution to avoid source confusion.

\subsection{Two Cautions}

It is important to recognize that even for those objects detected in SPIRE, the SPIRE data cannot be used to reliably estimate ISM masses (along the lines as done here) for the $z=1$ and 2 samples. For those redshifts, the SPIRE data will be probing near the rest-frame FIR luminosity peak-not safely on the RJ tail and not necessarily optically thin. The longest wavelength channel $(500 \mu \mathrm{m}$ or $600 \mathrm{GHz})$ will be probing restframe $170 \mu \mathrm{m}$ for $z=2$; for such measurements, there will be substantial uncertainty in the mass estimate, depending on the assumed value of the dust temperature (see Figure 2).

Fitting the observed SED to derive an effective dust temperature is not a reliable approach-near the FIR peak, the temperature characterizing the emission is "luminosity-weighted" (i.e., grains undergoing strong radiative heating) rather than massweighted. Hence, the derived $T_{d}$ will not reflect the temperature appropriate to the bulk of the ISM mass. Or, put another way, the flux measured near the peak is simply a measure of luminosity-not mass. Furthermore, the large SPIRE beam results in severe source confusion at these flux levels and hence unreliable flux measurements for individual galaxies. At $z>2$, ALMA resolution and sensitivity are required and one must observe at $v \leqslant 350 \mathrm{GHz}$ to be on the RJ tail of the dust emission.

\section{COSMOS MASS-SELECTED GALAXY SAMPLE FOR ALMA}

Our sample of 107 galaxies is taken from the COSMOS $2 \mathrm{deg}^{2}$ survey (Scoville et al. 2007), which has excellent photometric redshifts derived from 34 band (ultraviolet (UV)-mid-IR) photometry. The COSMOS field also has deep IR coverage with Spitzer (IRAC and MIPS) and Herschel (PACS and SPIRE), as well as radio continuum and X-ray coverage (Ilbert et al. 2013).

The galaxies were selected to have stellar masses $M_{*} \simeq$ $10^{11} M_{\odot}$, as determined from the photometric redshift SED template fit. The photometric redshifts and stellar masses of the galaxies are from Ilbert et al. (2013) and the accuracy of those quantities is discussed in detail there. The SFRs are derived from the rest-frame UV continuum and the IR using Herschel PACS and SPIRE data, as detailed in Scoville et al. (2013). For half of the objects, there exist reliable (multi-line) spectroscopic redshifts. 


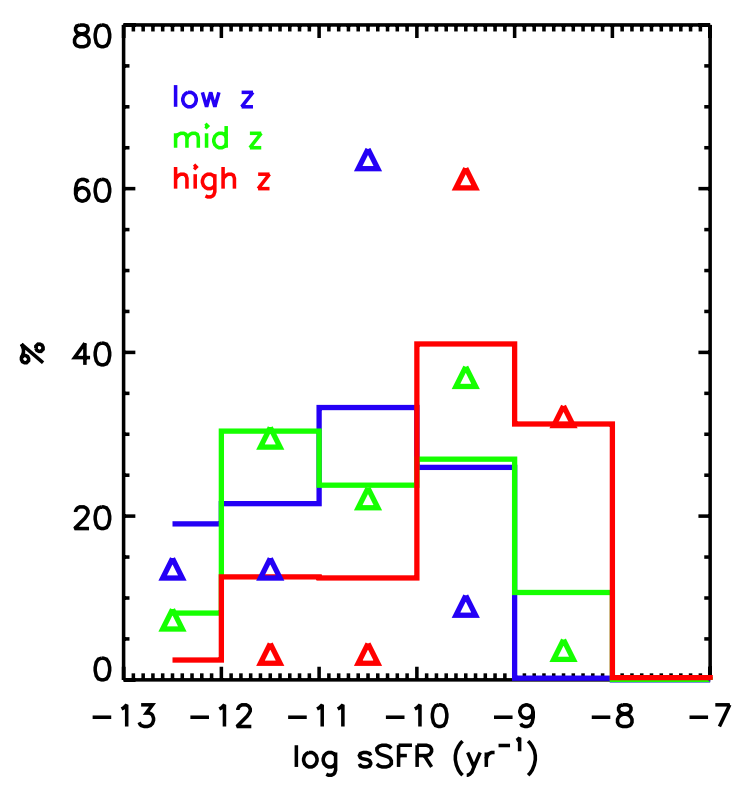

Figure 5. Distribution of specific SFRs are shown for the three redshift samples. The histograms show the distributions obtained from the full COSMOS photometric redshift catalog using the same redshift ranges and stellar mass ranges as observed here. The triangle points are the distributions for the three galaxy samples observed here.

(A color version of this figure is available in the online journal.)

In order to span the range of SF activity for massive galaxies at each redshift, the galaxies were selected to span the range of near-UV $-r(N U V-r)$ colors at each redshift. In the lower redshift samples, an increasingly large fraction of the objects would be classified as "passive" based on their red NUV- $r$ colors. Figure 5 shows the distributions of specific SFRs $\left(\mathrm{sSFR}=\mathrm{SFR} / M_{*}\right.$ ) for the full COSMOS galaxy samples and for the samples observed here.

The properties of the individual galaxies and their ALMA flux measurements and upper limits are listed in Tables 5-7 in the Appendix. The observed galaxies fall into four subsamples, all stellar mass-selected but over a range of redshifts. Their summary properties are given in Table 3 , including the median stellar mass $\left(M_{*}\right)$ and SFR of each sample. The first three samples were chosen based purely on stellar mass with no prior selection for either SFR or FIR luminosity. The last sample was specifically chosen to be IR-bright (easily detected by Herschel) and to have stellar masses $\sim 10^{11} M_{\odot}$, like the other samples.

\section{OBSERVATIONS AND RESULTS}

The ALMA Cycle 0 observations for project (\#2011.0. 00097.S) analyzed here were obtained in 2012 March-October in Band 7 at $350 \mathrm{GHz}$. On-source integration times were 1, 2, and 4 minutes per galaxy at $z=0.3,0.9$, and 2 , respectively. With continuum bandwidths of $8 \mathrm{GHz}$, the $1 \sigma \mathrm{rms}$ sensitivity was $0.5,0.4$, and $0.3 \mathrm{mJy}$ and typical synthesized beam sizes were $\simeq 0$.' 6 . The data were calibrated and imaged with natural weighting using CASA.

\subsection{Source Measurement and Noise Estimation}

The measurement results for the individual galaxies are tabulated in Tables 5-7. In the images, apertures centered on the galaxy position were used to search for significant detection of continuum in the integrated aperture flux ( $S_{\text {tot }}$ in the tables) or a high single pixel ( $S_{\text {peak }}$ in the tables) within the aperture. The former recovers instances where the emission is significantly
Table 3

Galaxy Samples for Dust Continuum Measurements

\begin{tabular}{lcccc}
\hline \hline Sample & No. & $z$ & $\begin{array}{c}\left\langle M_{\text {stellar }}\right\rangle^{\mathrm{a}} \\
\left(10^{11} M_{\odot}\right)\end{array}$ & $\begin{array}{l}\log \langle\mathrm{SFR}\rangle^{\mathrm{a}} \\
\left(M_{\odot} \mathrm{yr}^{-1}\right)\end{array}$ \\
\hline Low- $z$ & 37 & $0.22-0.48$ & $1.30 \pm 0.57$ & $0.63 \pm 1.72$ \\
Mid- $z$ & 33 & $0.81-1.15$ & $1.59 \pm 0.66$ & $1.31 \pm 1.68$ \\
High- $z$ & 31 & $1.46-2.54$ & $1.23 \pm 0.61$ & $1.97 \pm 0.57$ \\
IR-bright & 6 & $1.46-2.02$ & $1.00 \pm 0.33$ & $2.27 \pm 0.23$ \\
\hline
\end{tabular}

Note. ${ }^{a}$ The uncertainties give the dispersion of each sample.

extended beyond the synthesized beam $\left(\simeq 1^{\prime \prime}\right)$; the latter is most sensitive when the emission is unresolved. The apertures were $3^{\prime \prime}$ and $2^{\prime \prime}$ for the low- $z$ sample and the mid- and high- $z$ samples, respectively. A significant detection required a $2 \sigma \mathrm{S} / \mathrm{N}$ in the integrated aperture flux ( $S_{\text {tot }}$ in the tables) or a $3.6 \sigma$ detection of a high single pixel ( $S_{\text {peak }}$ in the tables) within the aperture. These different n $\sigma$ limits were chosen such that there would be fewer than one spurious detection by either technique in the entire sample.

The noise estimate in both cases was derived from the dispersion in the integrated and peak flux measurements obtained for 100 apertures of the same size displaced off-center in the same image. We also tabulate the $\mathrm{S} / \mathrm{Ns}$ given by

$$
\begin{aligned}
\mathrm{S} / \mathrm{N}_{\text {tot }} & =\frac{S_{\text {tot }}}{\sigma_{\text {tot }}} \\
\mathrm{S} / \mathrm{N}_{\text {peak }} & =\frac{S_{\text {peak }}}{\sigma_{\text {pix }}} .
\end{aligned}
$$

Note that we let the $\mathrm{S} / \mathrm{N}$ be less than 0 if the measured flux is less than 0 ; this is so that large negative flux values do not end up with a positive $\mathrm{S} / \mathrm{N}$ (above the detection thresholds).

The $\mathrm{S} / \mathrm{N}$ given in Column 9 (Tables 5-7) is the better of those obtained from the integrated or peak flux measurement. In the last column, limits are given at $2 \sigma$ and $3.6 \sigma$ in the inferred mass, depending on whether the better $\mathrm{S} / \mathrm{N}$ was obtained for the integrated or peak flux measurement.

Of the 107 galaxies, 28 (26\%) were significantly detected and these are shown in Figure 9. The maximum detected $350 \mathrm{GHz}$ flux was $\sim 4 \mathrm{mJy}$ and the inferred ISM masses are $1-8 \times 10^{10} \mathrm{M}_{\odot}$. Although the detection rate is less than $50 \%$, one should recall that the integration times were only 1-4 minutes with fewer than 18 of the eventual 64 ALMA telescopes. Second, this very high stellar mass sample will preferentially include a large fraction of passive galaxies and the ISM contents may be larger at lower stellar mass. The detection rate was $100 \%$ for the IR-bright sample.

\section{STACKED SAMPLES}

The mean fluxes and derived ISM masses for stacked images of each of the four galaxy subsamples may be used to explore the overall evolution of the dust emission and ISM masses. The individual galaxy images were stacked in three ways: an average, a median stack, and an average weighted by the square of the rms noise. The median and weighted averages are used in the discussion and figures below. (The simple average was not significantly different except that it has higher noise than the rms-weighted average.) Figure 6 shows the median stacked images for each of these subsamples.

Figure 7 shows the average and median $350 \mathrm{GHz}$ fluxes for each of the four stacked samples. Table 4 lists these 

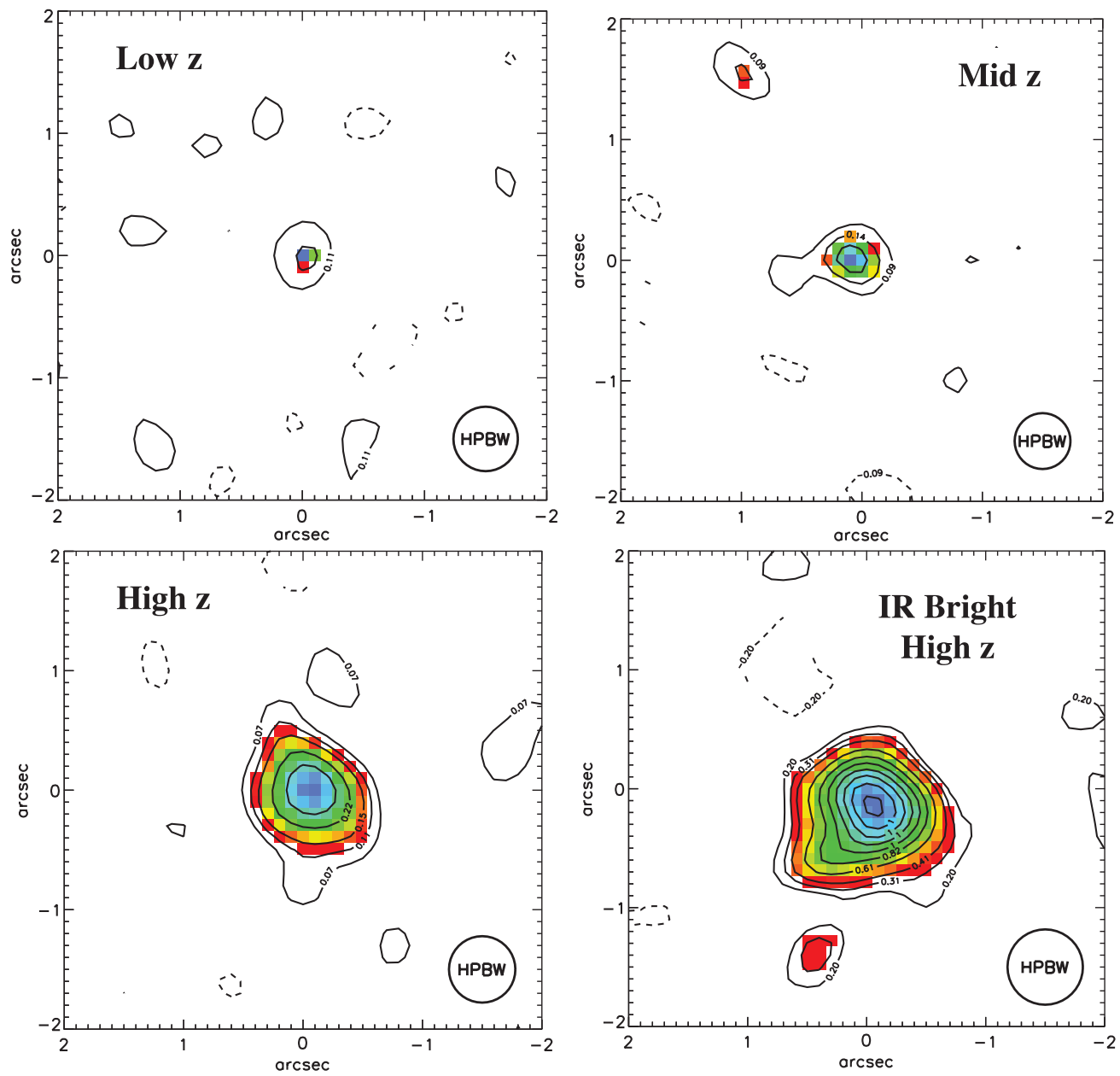

Figure 6. Median stacked images for the four subsamples of galaxies (Tables 3 and 4). The scale is in arcsecs and the contours are at $-2,2,3,4,5,7,9$, and $11 \sigma$ (see Table 4).

(A color version of this figure is available in the online journal.)

Table 4

Stacked Samples

\begin{tabular}{|c|c|c|c|c|c|c|c|c|c|c|}
\hline Stack & $\begin{array}{c}S v \\
(\mathrm{mJy})\end{array}$ & $\begin{array}{c}\sigma_{\text {pix }} \\
(\mathrm{mJy})\end{array}$ & $\begin{array}{l}\sigma_{\text {total }} \\
(\mathrm{mJy})\end{array}$ & $\mathrm{S} / \mathrm{N}^{\mathrm{a}}$ & $\langle z\rangle$ & $\begin{array}{c}\left\langle M_{*}\right\rangle \\
\left(10^{11} M_{\odot}\right) \\
\end{array}$ & $\begin{array}{l}\log \langle\mathrm{SFR}\rangle \\
\left(M_{\odot} \mathrm{yr}^{-1}\right) \\
\end{array}$ & $\begin{array}{c}\left\langle M_{\text {ISM }}\right\rangle \\
\left(10^{10} M_{\odot}\right) \\
\end{array}$ & $\begin{array}{l}\left\langle\tau_{\mathrm{SF}}\right\rangle \\
(\mathrm{Gyr})\end{array}$ & $M_{\mathrm{ISM}} /\left(M_{*}+M_{\mathrm{ISM}}\right)$ \\
\hline \multicolumn{11}{|c|}{ Low $-z$} \\
\hline Weighted average & 0.18 & 0.057 & 0.240 & 3.23 & 0.39 & $1.30 \pm 0.57$ & $0.63 \pm 1.72$ & $0.21 \pm 0.07$ & $0.50 \pm 0.16$ & $0.02 \pm 0.005$ \\
\hline Median & 0.22 & 0.073 & 0.280 & 3.55 & 0.37 & $1.11 \pm 0.57$ & $-0.13 \pm 1.72$ & $0.25 \pm 0.07$ & $0.64 \pm 0.18$ & $0.02 \pm 0.006$ \\
\hline \multicolumn{11}{|c|}{ Mid- $z$} \\
\hline Weighted average & 0.88 & 0.045 & 0.245 & 4.63 & 0.89 & $1.59 \pm 0.66$ & $1.31 \pm 1.68$ & $2.19 \pm 0.47$ & $1.06 \pm 0.23$ & $0.12 \pm 0.026$ \\
\hline Median & 0.88 & 0.059 & 0.274 & 3.51 & 0.87 & $1.41 \pm 0.66$ & $0.10 \pm 1.68$ & $2.20 \pm 0.63$ & $1.15 \pm 0.33$ & $0.13 \pm 0.038$ \\
\hline \multicolumn{11}{|c|}{ High- $z$} \\
\hline Weighted average & 0.75 & 0.037 & 0.108 & 9.96 & 2.07 & $1.23 \pm 0.61$ & $1.97 \pm 0.57$ & $2.43 \pm 0.24$ & $0.26 \pm 0.03$ & $0.16 \pm 0.017$ \\
\hline Median & 0.50 & 0.047 & 0.117 & 6.72 & 1.98 & $1.09 \pm 0.61$ & $1.78 \pm 0.57$ & $1.60 \pm 0.24$ & $0.16 \pm 0.02$ & $0.13 \pm 0.019$ \\
\hline \multicolumn{11}{|c|}{ IR bright } \\
\hline Weighted average & 3.36 & 0.102 & 0.249 & 18.44 & 1.66 & $1.00 \pm 0.33$ & $2.27 \pm 0.23$ & $10.48 \pm 0.57$ & $0.57 \pm 0.03$ & $0.51 \pm 0.028$ \\
\hline Median & 3.82 & 0.130 & 0.286 & 15.42 & 1.70 & $0.93 \pm 0.33$ & $2.22 \pm 0.23$ & $11.91 \pm 0.77$ & $0.54 \pm 0.04$ & $0.56 \pm 0.036$ \\
\hline
\end{tabular}

Notes. For the mid- $z$ and high- $z$ stacks, the total aperture flux was used. For the low- $z$ stack, the flux is from the peak pixel flux within the $3^{\prime \prime}$ aperture. This was necessitated since the lower signal strength did not allow a significant detection in the integrated aperture flux. $\tau_{\mathrm{SF}}=M_{\mathrm{ISM}} /\langle\mathrm{SFR}\rangle$ where $\langle\mathrm{SFR}\rangle$ is the mean SFR. The uncertainties given for each quantity are the standard deviations.

${ }^{a} \mathrm{~S} / \mathrm{N}_{\text {tot }}$ and $\mathrm{S} / \mathrm{N}_{\text {peak }}$ calculated separately from Equation (14) and the $\mathrm{S} / \mathrm{N}$ column lists the larger in absolute magnitude of those two $\mathrm{S} / \mathrm{Ns}$. 


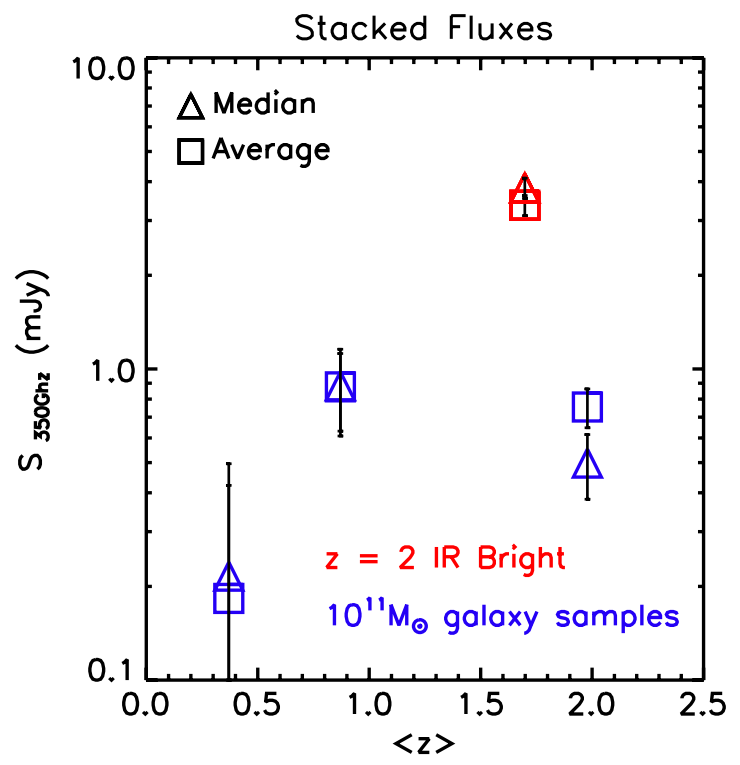

Figure 7. $350 \mathrm{GHz}$ continuum fluxes from stacked images of the four galaxies samples. Median and average stacked images are indicated by triangles and squares. The $\mathrm{S} / \mathrm{Ns}$ are given in Table 4 and they are from $\sim 3,4,8$, and 16 for the low- $z$, mid- $z$, high- $z$, and IR-bright samples, respectively.

(A color version of this figure is available in the online journal.)

measurements along with their S/N. All four samples are significantly detected in the stacked images (both median and average). The lowest redshift sample does not have high statistical significance in the stacked detection, but that is to be expected, given the anticipated evolution of ISM masses for these very massive galaxies.

The derived ISM masses for each of the samples are shown in Figure 8 for the average and median stacks. The figures clearly show an increase in the ISM content both in absolute mass and gas mass fraction (since the stellar masses are the same) from $z=0.4$ to 1 . From $z=1$ to 2 , the ISM mass shows no significant evolution for the strictly stellar mass-selected samples. This is a very interesting result, but given the small sample sizes and the fact that these are only the most massive galaxies, it is not clear how general these results are in terms of overall cosmic evolution. A high priority should be placed on extending the samples to lower stellar mass and better sampling of the SF characteristics.

Comparing the IR-bright sample at $z=2$ with the strictly mass-selected sample at the same redshift, it is clear that the IR-bright galaxies have considerably higher long wavelength fluxes and presumably higher ISM contents by a factor $\sim 5$. This increase in the apparent ISM mass is in fact larger than the increase in the estimated SFRs. If this holds up, it would clearly suggest that the $z=2$ IR-bright galaxies are extra luminous simply because they have more ISM.

The depletion timescales for the ISM via SF are given by $\tau_{\mathrm{SF}}=M_{\mathrm{ISM}} /\langle\mathrm{SFR}\rangle$ and are also listed in Table 4 . This depletion timescale is similar for the subsamples $(0.2-1.2 \mathrm{Gyr})$. Both of the $z \sim 2$ samples have similar depletion timescales $(0.2$ and 0.5 Gyr for the normal and IR-bright samples, respectively). This underscores the point made above that the IR-bright galaxies simply have more ISM, rather than having a higher SF efficiency.

The gas mass fractions $\left(M_{\mathrm{ISM}} /\left(M_{*}+M_{\mathrm{ISM}}\right)\right)$ are $\sim 2 \% \pm$ $0.5 \%, 12 \% \pm 3 \%, 14 \% \pm 3 \%$, and $53 \% \pm 5 \%$ for the low $-z$, mid- $z$, high- $z$, and IR-bright samples, respectively. Tacconi et al. (2013) surveyed 52 galaxies at $z=1-3$ in $\mathrm{CO}(3-2)$ and found mean gas mass fractions of 0.33 and 0.47 at $z \sim 1.2$ and 2.2, respectively. Their sample was selected with $M_{*}>2.5 \times 10^{10} M_{\odot}$ and SFR $>32 M_{\odot} \mathrm{yr}^{-1}$; it is therefore likely to favor more active star-forming galaxies than our purely stellar-mass-selected samples. On the other hand, our IR-bright sample is likely to be more star forming than their average galaxy so it is entirely reasonable to find a somewhat higher gas mass fraction.

\section{SUMMARY}

We have developed the physical and empirical basis for using the long wavelength RJ dust emission as an accurate and fast probe of the ISM content of galaxies.
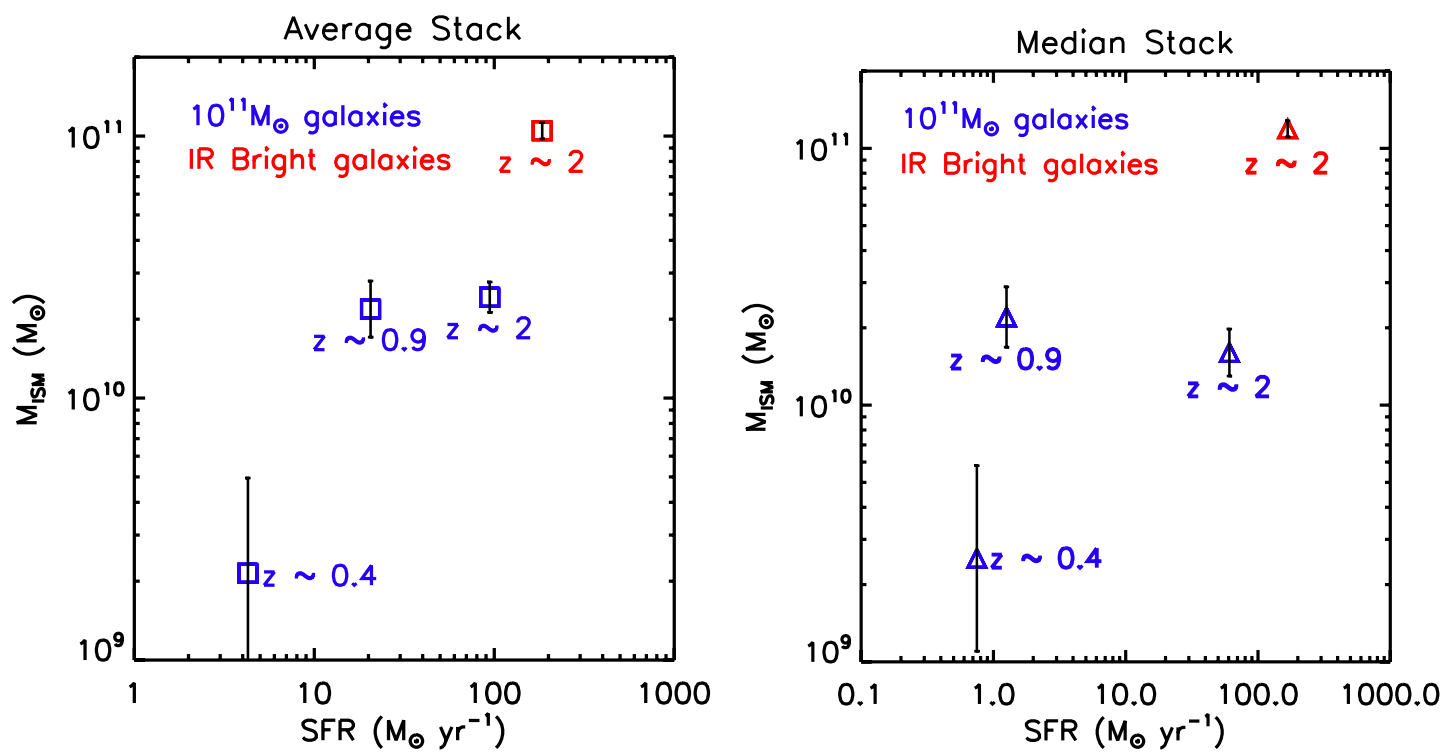

Figure 8. Derived ISM masses of the average (left) and median (right) stack images are shown with their median and average SFRs. The SF timescales $\left(\tau_{\mathrm{SF}}=M_{\mathrm{ISM}} /\langle\mathrm{SFR}\rangle\right)$, listed in Table 4 , are in the range $0.2-1.2 \mathrm{Gyr}$.

(A color version of this figure is available in the online journal.) 
Table 5

Low- $z$ Galaxy Sample in Band 7

\begin{tabular}{|c|c|c|c|c|c|c|c|c|c|c|c|}
\hline No. & $\begin{array}{c}\text { R.A. }(2000) \\
\left({ }^{\circ}\right)\end{array}$ & $\begin{array}{c}\text { Decl. (2000) } \\
\left({ }^{\circ}\right)\end{array}$ & $z^{\mathrm{a}}$ & $\begin{array}{c}S_{\mathrm{tot}} \\
(\mathrm{mJy})\end{array}$ & $\begin{array}{c}\sigma_{\text {tot }} \\
(\mathrm{mJy})\end{array}$ & $\begin{array}{c}S_{\text {peak }} \\
(\mathrm{mJy})\end{array}$ & $\begin{array}{c}\sigma_{\text {pix }} \\
(\mathrm{mJy})\end{array}$ & $\mathrm{S} / \mathrm{N}^{\mathrm{b}}$ & $\begin{array}{c}M_{*}{ }^{\mathrm{a}} \\
\left(10^{11} M_{\odot}\right)\end{array}$ & $\begin{array}{c}\log (\mathrm{SFR})^{\mathrm{a}} \\
\left(M_{\odot} \mathrm{yr}^{-1}\right)\end{array}$ & $\begin{array}{c}M_{\mathrm{ISM}} \\
\left(10^{10} M_{\odot}\right)\end{array}$ \\
\hline \multicolumn{12}{|c|}{ Low- $z$} \\
\hline 1 & 150.2142 & 1.7578 & 0.47 & -0.06 & 1.57 & 0.91 & 0.44 & -0.04 & 1.22 & -3.00 & $<1.98$ \\
\hline 2 & 150.0296 & 1.7375 & 0.28 & -1.66 & 1.60 & 1.07 & 0.39 & -1.04 & 0.71 & -3.00 & $<0.95$ \\
\hline 3 & 149.7943 & 1.7293 & 0.35 & 0.37 & 1.57 & 0.99 & 0.38 & 0.23 & 0.89 & 0.76 & $<1.21$ \\
\hline 4 & 150.4186 & 1.8580 & 0.46 & -1.92 & 1.18 & 0.73 & 0.27 & -1.63 & 0.86 & -1.60 & $<1.17$ \\
\hline 5 & 150.0034 & 1.9173 & 0.27 & 0.85 & 1.04 & 1.05 & 0.42 & 0.82 & 1.40 & -3.00 & $<0.96$ \\
\hline 6 & 149.8612 & 1.8387 & 0.44 & 1.29 & 1.95 & 1.05 & 0.45 & 0.66 & 1.71 & -3.00 & $<1.86$ \\
\hline 7 & 149.6918 & 1.8285 & 0.46 & 2.17 & 1.81 & 1.27 & 0.41 & 1.20 & 1.00 & 1.54 & $<1.79$ \\
\hline 8 & 150.5417 & 2.7316 & 0.35 & 1.39 & 0.74 & 0.68 & 0.29 & 1.88 & 3.42 & -3.00 & $<0.93$ \\
\hline 9 & 150.6447 & 1.9532 & 0.45 & 1.54 & 0.93 & 0.74 & 0.26 & 1.65 & 1.78 & -3.00 & $<1.09$ \\
\hline 10 & 150.3202 & 2.0114 & 0.31 & -1.59 & 1.39 & 1.53 & 0.48 & -1.15 & 0.67 & -2.71 & $<1.33$ \\
\hline 11 & 150.2115 & 2.0629 & 0.37 & -0.84 & 2.09 & 1.54 & 0.49 & -0.40 & 0.79 & -3.00 & $<1.68$ \\
\hline 12 & 149.6316 & 2.0700 & 0.31 & -0.81 & 2.19 & 0.98 & 0.43 & -0.37 & 0.65 & -2.40 & $<1.18$ \\
\hline 13 & 150.5090 & 2.0401 & 0.37 & 2.07 & 1.05 & 0.83 & 0.28 & 1.98 & 0.90 & -3.00 & $<0.97$ \\
\hline 14 & 150.0951 & 2.1937 & 0.42 & -0.08 & 0.89 & 0.65 & 0.27 & -0.09 & 1.12 & -0.10 & $<1.07$ \\
\hline 15 & 149.6461 & 1.8434 & 0.37 & -2.84 & 1.97 & 1.11 & 0.42 & -1.44 & 0.56 & 0.40 & $<1.43$ \\
\hline 16 & 150.7706 & 2.3259 & 0.45 & -4.93 & 1.07 & 0.62 & 0.29 & -4.62 & 1.97 & -1.14 & $<1.24$ \\
\hline 17 & 150.1714 & 2.2379 & 0.34 & -1.06 & 2.44 & 1.08 & 0.45 & -0.43 & 1.00 & 0.95 & $<1.40$ \\
\hline 18 & 149.6073 & 2.6646 & 0.36 & -0.60 & 1.22 & 0.82 & 0.38 & -0.49 & 2.24 & 1.16 & $<1.26$ \\
\hline 19 & 149.9209 & 2.0312 & 0.36 & 2.36 & 1.84 & 1.25 & 0.41 & 1.28 & 1.26 & 0.96 & $<1.32$ \\
\hline 20 & 149.8058 & 2.2894 & 0.47 & -0.06 & 1.57 & 0.99 & 0.43 & -0.04 & 0.89 & 0.20 & $<1.92$ \\
\hline 21 & 150.1480 & 2.3108 & 0.48 & 3.48 & 1.60 & 1.23 & 0.47 & 2.17 & 1.12 & 0.10 & $5.30 \pm 2.44$ \\
\hline 22 & 150.5859 & 2.3711 & 0.43 & 1.43 & 0.99 & 0.77 & 0.30 & 1.45 & 1.07 & -2.31 & $<1.20$ \\
\hline 23 & 150.1541 & 2.2262 & 0.35 & 0.66 & 1.16 & 1.09 & 0.44 & 0.57 & 0.92 & 0.53 & $<1.40$ \\
\hline 24 & 150.0049 & 2.5036 & 0.34 & 0.73 & 1.60 & 1.40 & 0.44 & 0.46 & 1.26 & 0.56 & $<1.35$ \\
\hline 25 & 150.5190 & 2.1952 & 0.37 & 0.30 & 1.09 & 1.06 & 0.28 & 3.81 & 0.71 & 0.91 & $0.97 \pm 0.26$ \\
\hline 26 & 150.0951 & 2.1937 & 0.42 & 0.58 & 1.59 & 1.15 & 0.41 & 0.36 & 1.12 & -0.10 & $<1.62$ \\
\hline 27 & 150.4988 & 2.0749 & 0.42 & 0.45 & 1.26 & 0.74 & 0.28 & 0.35 & 1.11 & 0.81 & $<1.10$ \\
\hline 28 & 150.5009 & 2.6119 & 0.34 & 0.45 & 1.07 & 0.73 & 0.28 & 0.42 & 0.89 & 0.59 & $<0.85$ \\
\hline 29 & 150.0294 & 1.9970 & 0.39 & 0.35 & 1.62 & 1.61 & 0.41 & 3.94 & 0.63 & -2.50 & $2.13 \pm 0.54$ \\
\hline 30 & 150.5183 & 2.3695 & 0.45 & -0.86 & 1.00 & 0.72 & 0.27 & -0.86 & 2.00 & 1.17 & $<1.15$ \\
\hline 31 & 150.3504 & 2.2755 & 0.47 & 1.75 & 1.15 & 0.65 & 0.28 & 1.51 & 1.55 & 1.18 & $<1.25$ \\
\hline 32 & 150.4906 & 2.5882 & 0.36 & 0.91 & 1.23 & 0.71 & 0.27 & 0.74 & 1.54 & -0.13 & $<0.88$ \\
\hline 33 & 150.1707 & 2.5669 & 0.41 & -0.87 & 1.08 & 0.98 & 0.41 & -0.81 & 1.13 & -2.61 & $<1.60$ \\
\hline 34 & 150.0071 & 1.7208 & 0.37 & 0.02 & 1.60 & 1.01 & 0.43 & 0.01 & 0.79 & -1.70 & $<1.48$ \\
\hline 35 & 150.3371 & 2.2795 & 0.35 & 0.28 & 1.00 & 0.78 & 0.25 & 0.28 & 1.58 & 1.02 & $<0.81$ \\
\hline 36 & 150.0943 & 2.1393 & 0.22 & 1.06 & 1.63 & 1.67 & 0.44 & 3.84 & 0.67 & -2.87 & $1.84 \pm 0.48$ \\
\hline 37 & 149.8424 & 2.3006 & 0.26 & 0.55 & 1.44 & 1.12 & 0.40 & 0.38 & 1.78 & -2.65 & $<0.89$ \\
\hline
\end{tabular}

Notes.

a The photometric redshifts and stellar masses of the galaxies are from Ilbert et al. (2013) and the accuracy of those quantities is discussed in detail there. The SFRs are derived from the rest-frame UV continuum and the IR using Herschel PACS and SPIRE data, as detailed in Scoville et al. (2013). All of the galaxies have greater than $10 \sigma$ photometry measurements so the uncertainties in $M_{*}$ and SFR associated with their measurements are always less than $10 \%$. As discussed in Ilbert et al. (2013), the uncertainties in models used to derive the $M_{*}$ and SFR from the photometry are generally much larger but generally less than a factor two.

${ }^{b} \mathrm{~S} / \mathrm{N}_{\text {tot }}$ and $\mathrm{S} / \mathrm{N}_{\text {peak }}$ calculated separately from Equation (14) and the column $\mathrm{S} / \mathrm{N}$ lists the larger in absolute magnitude of those two S/Ns. Note that we let the $\mathrm{S} / \mathrm{N}$ be negative in cases where the flux estimate is negative so that several sigma negative flux values do not end up with a positive $\mathrm{S} / \mathrm{N}$ above the detection thresholds.

To obviate the need to know both the dust opacity and dustto-gas ratio (which are degenerate in Equation (1)), we have chosen instead to empirically calibrate the ratio of the specific luminosity at rest frame $850 \mu \mathrm{m}$ to the total ISM mass using samples of observed galaxies, thus absorbing the opacity curve and the abundance ratio into a single empirical constant $\alpha_{850 \mu \mathrm{m}}=L_{\nu_{850 \mu \mathrm{m}}} / M_{\text {ISM }}$. Three samples were developed: (1) 12 local star-forming and star-bursting galaxies with global SCUBA and ISM measurements, (2) extensive Galactic observations from the Planck Collaboration, and (3) a sample of 28 SMGs at $z<3$ with $\mathrm{CO}(1-0)$ measurements. The three samples yielded $\alpha_{850 \mu \mathrm{m}}=1.0,0.79$, and $1.01 \times 10^{20} \mathrm{erg} \mathrm{s}^{-1} \mathrm{~Hz}^{-1} M_{\odot}{ }^{-1}$, respectively.
The Planck measurements are particularly convincing-they are of high $\mathrm{S} / \mathrm{N}$, span a large wavelength range. and they probe the diversity of Galactic ISM including both $\mathrm{HI}$ - and $\mathrm{H}_{2}$-dominated clouds, exhibiting little variation in the empirical $\alpha_{250 \mu \mathrm{m}}$ (Planck Collaboration 2011b). The Planck measurements also determine very well the long wavelength dust emissivity index: $\beta=1.8 \pm 0.1$ (Planck Collaboration 2011a).

In our ALMA observations, we intentionally sample high stellar mass galaxies that should have close to solar metallicity (Erb et al. 2006) in order to avoid the issue of possible variations in dust-to-gas ratios at low metallicity. However, we note that Draine et al. (2007) see no variation in the dust abundance down to $\sim 20 \%$ of solar metallicity and it is reassuring that the low- $z$ 
Table 6

Mid- $z$ Galaxy Sample in Band 7

\begin{tabular}{|c|c|c|c|c|c|c|c|c|c|c|c|}
\hline No. & $\begin{array}{c}\text { R.A. (2000) } \\
\left({ }^{\circ}\right)\end{array}$ & $\begin{array}{c}\text { Decl. (2000) } \\
\left({ }^{\circ}\right)\end{array}$ & $z^{\mathrm{a}}$ & $\begin{array}{c}S_{\mathrm{tot}} \\
(\mathrm{mJy})\end{array}$ & $\begin{array}{c}\sigma_{\text {tot }} \\
(\mathrm{mJy})\end{array}$ & $\begin{array}{l}S_{\text {peak }} \\
(\mathrm{mJy})\end{array}$ & $\begin{array}{c}\sigma_{\text {pix }} \\
(\mathrm{mJy})\end{array}$ & $\mathrm{S} / \mathrm{N}^{\mathrm{b}}$ & $\begin{array}{c}M_{*}^{\mathrm{a}} \\
\left(10^{11} M_{\odot}\right)\end{array}$ & $\begin{array}{c}\log (\mathrm{SFR})^{\mathrm{a}} \\
\left(M_{\odot} \mathrm{yr}^{-1}\right)\end{array}$ & $\begin{array}{c}M_{\mathrm{ISM}} \\
\left(10^{10} M_{\odot}\right)\end{array}$ \\
\hline \multicolumn{12}{|c|}{ Mid- $z$} \\
\hline 1 & 150.0728 & 1.7361 & 0.84 & 1.76 & 1.86 & 1.27 & 0.42 & 0.95 & 2.18 & -0.67 & $<3.06$ \\
\hline 2 & 149.8392 & 2.2262 & 0.94 & 0.88 & 1.49 & 1.20 & 0.43 & 0.59 & 0.89 & -0.60 & $<3.32$ \\
\hline 3 & 149.7302 & 2.0708 & 0.88 & 1.93 & 1.15 & 0.66 & 0.23 & 1.68 & 0.65 & -1.04 & $<1.72$ \\
\hline 4 & 150.1133 & 2.0142 & 0.84 & -0.12 & 0.90 & 0.56 & 0.21 & -0.13 & 0.87 & -3.00 & $<1.53$ \\
\hline 5 & 150.5422 & 2.2772 & 0.87 & -1.61 & 1.03 & 0.32 & 0.21 & -1.56 & 1.55 & -0.78 & $<1.56$ \\
\hline 6 & 150.0771 & 2.5490 & 0.88 & -2.12 & 1.46 & 1.17 & 0.47 & -1.45 & 1.41 & -0.40 & $<3.50$ \\
\hline 7 & 150.4510 & 2.4353 & 0.99 & -0.38 & 0.66 & 0.59 & 0.21 & -0.58 & 3.06 & -1.11 & $<1.64$ \\
\hline 8 & 149.6546 & 2.7470 & 0.84 & -0.01 & 0.72 & 0.49 & 0.16 & -0.01 & 3.16 & -3.00 & $<1.19$ \\
\hline 9 & 150.6991 & 2.3539 & 0.98 & 0.80 & 0.85 & 0.64 & 0.22 & 0.95 & 1.45 & -3.00 & $<1.70$ \\
\hline 10 & 150.4478 & 1.8505 & 0.87 & 0.65 & 0.74 & 0.55 & 0.21 & 0.88 & 1.23 & -3.00 & $<1.59$ \\
\hline 11 & 150.3820 & 2.1208 & 1.15 & -0.23 & 0.83 & 0.50 & 0.20 & -0.28 & 2.02 & -3.00 & $<1.69$ \\
\hline 12 & 150.3798 & 2.0147 & 0.83 & 0.59 & 0.72 & 0.60 & 0.21 & 0.82 & 0.91 & 0.70 & $<1.53$ \\
\hline 13 & 149.8340 & 1.9810 & 0.94 & -1.18 & 1.39 & 1.17 & 0.43 & -0.85 & 2.00 & 0.40 & $<3.33$ \\
\hline 14 & 150.4944 & 2.2822 & 0.84 & 0.19 & 0.82 & 0.45 & 0.22 & 0.23 & 1.56 & -3.00 & $<1.59$ \\
\hline 15 & 149.7526 & 2.2140 & 1.01 & 1.78 & 0.85 & 0.61 & 0.22 & 2.11 & 1.14 & 2.26 & $4.77 \pm 2.26$ \\
\hline 16 & 150.2639 & 1.9714 & 0.93 & -0.57 & 0.65 & 0.46 & 0.20 & -0.87 & 1.73 & 1.65 & $<1.57$ \\
\hline 17 & 149.9631 & 2.6131 & 0.90 & -1.37 & 1.94 & 0.92 & 0.41 & -0.71 & 0.89 & 0.90 & $<3.08$ \\
\hline 18 & 150.5358 & 2.2553 & 0.93 & 2.62 & 0.83 & 1.00 & 0.19 & 5.17 & 0.56 & 1.74 & $2.41 \pm 0.47$ \\
\hline 19 & 149.7205 & 2.7103 & 0.88 & 2.96 & 0.99 & 1.06 & 0.23 & 4.70 & 1.78 & 1.60 & $2.62 \pm 0.56$ \\
\hline 20 & 149.8618 & 2.1731 & 0.86 & 3.94 & 1.99 & 1.94 & 0.40 & 4.90 & 1.41 & 1.81 & $4.97 \pm 1.01$ \\
\hline 21 & 149.7728 & 1.8542 & 0.82 & -0.50 & 1.63 & 0.79 & 0.41 & -0.31 & 1.41 & 1.51 & $<2.96$ \\
\hline 22 & 149.8820 & 2.3506 & 0.86 & -0.72 & 1.39 & 1.15 & 0.41 & -0.52 & 0.88 & 1.42 & $<3.06$ \\
\hline 23 & 150.0685 & 2.2655 & 0.97 & 1.71 & 2.02 & 1.18 & 0.44 & 0.84 & 1.27 & 1.55 & $<3.47$ \\
\hline 24 & 149.9149 & 2.0944 & 0.85 & 0.06 & 1.88 & 1.22 & 0.43 & 0.03 & 1.14 & 0.21 & $<3.15$ \\
\hline 25 & 150.1289 & 2.1932 & 0.87 & 1.60 & 0.72 & 0.54 & 0.20 & 2.22 & 2.23 & 1.37 & $3.95 \pm 1.78$ \\
\hline 26 & 150.1089 & 2.0116 & 0.87 & 3.67 & 1.21 & 0.96 & 0.36 & 3.02 & 0.71 & 0.20 & $9.05 \pm 3.00$ \\
\hline 27 & 150.6456 & 1.9368 & 0.82 & -0.18 & 0.76 & 0.50 & 0.19 & -0.24 & 0.63 & 0.10 & $<1.37$ \\
\hline 28 & 149.7644 & 2.3401 & 0.81 & 1.04 & 0.93 & 0.69 & 0.23 & 1.11 & 1.78 & 1.92 & $<1.63$ \\
\hline 29 & 150.0906 & 2.5505 & 0.87 & -0.01 & 1.43 & 0.61 & 0.33 & -0.01 & 1.83 & -0.11 & $<2.42$ \\
\hline 30 & 149.9190 & 2.6430 & 0.88 & 1.27 & 1.30 & 1.08 & 0.42 & 0.98 & 0.54 & -0.67 & $<3.16$ \\
\hline 31 & 150.4074 & 1.8032 & 0.82 & 0.99 & 0.54 & 0.48 & 0.21 & 1.82 & 1.78 & 1.32 & $<1.51$ \\
\hline 32 & 149.9986 & 2.0634 & 0.91 & 1.76 & 0.84 & 1.39 & 0.39 & 2.09 & 1.26 & -0.00 & $4.46 \pm 2.13$ \\
\hline 33 & 150.2983 & 2.0421 & 0.83 & 0.81 & 0.66 & 0.56 & 0.21 & 1.22 & 2.24 & -0.50 & $<1.53$ \\
\hline
\end{tabular}

Notes.

a The photometric redshifts and stellar masses of the galaxies are from Ilbert et al. (2013) and the accuracy of those quantities is discussed in detail there. The SFRs are derived from the rest-frame UV continuum and the IR using Herschel PACS and SPIRE data, as detailed in Scoville et al. (2013). All of the galaxies have greater than $10 \sigma$ photometry measurements so the uncertainties in $M_{*}$ and SFR associated with their measurements are always less than $10 \%$. As discussed in Ilbert et al. (2013), the uncertainties in models used to derive the $M_{*}$ and SFR from the photometry are generally much larger but generally less than a factor two.

b $\mathrm{S} / \mathrm{N}_{\text {tot }}$ and $\mathrm{S} / \mathrm{N}_{\text {peak }}$ calculated separately from Equation (14) and the column $\mathrm{S} / \mathrm{N}$ lists the larger in absolute magnitude of those two $\mathrm{S} / \mathrm{Ns}$. Note that we let the $\mathrm{S} / \mathrm{N}$ be negative in cases where the flux estimate is negative so that several sigma negative flux values do not end up with a positive $\mathrm{S} / \mathrm{N}$ above the detection thresholds.

sample and the high- $z$ SMGs yield similar $\alpha_{850 \mu \mathrm{m}}$. Last, we note that the consistency of all these calibrations (nearby galaxies, the Milky Way, and high- $z$ SMGs) is strongly suggestive that the CO-to-H 2 conversion factor $\left(\alpha_{\mathrm{CO}}\right)$ does not vary greatly when applied to global ISM measurements.

We then applied the derived calibrations to our ALMA dust continuum observations for a sample of 107 galaxies in COSMOS with stellar mass $M_{*} \simeq 10^{11} M_{\odot}$ at three redshifts: $z=0.4,0.9$, and 2. Strong evolution is seen with the ISM mass fraction, decreasing from $53 \% \pm 5 \%$ of the total (ISM+stars) mass at the higher redshifts down to $\sim 2 \% \pm 0.5 \%$ at the lowest redshift. In addition, a small sample of 6 IR-luminous galaxies at $z=2$ has $\sim 5$ times greater ISM mass than the equivalent non-IR-bright sample at the same redshift.

Although these preliminary observations detect only a minority of the individual objects, the mean fluxes and masses obtained from the stacked data will be most worthwhile in providing a priori estimates for expected fluxes in future observational programs. Thus, required sensitivity and time estimates are more reliable and sample sizes can be chosen appropriately based on the fluxes presented in Figure 3.

This was a "pathfinder" study using ALMA in Cycle 0 with just $5 \mathrm{hr}$ of time in Band $7(350 \mathrm{GHz})$ and a maximum of 18 telescopes. Thus, the evolutionary trends mentioned above are very preliminary and much larger samples are warranted. It should be emphasized that the technique advocated here is both much faster (by at least a factor 10) than using the standard molecular line tracers such as CO; it is also very likely more robust - avoiding the uncertainties of variable molecular excitation and the need to observe higher rotational transitions at high redshift.

Last, we note that using the dust continuum to probe the ISM content does not require high accuracy spectroscopic redshifts and may instead be done on much larger samples 
Table 7

High- $z$ Galaxy Sample in Band 7

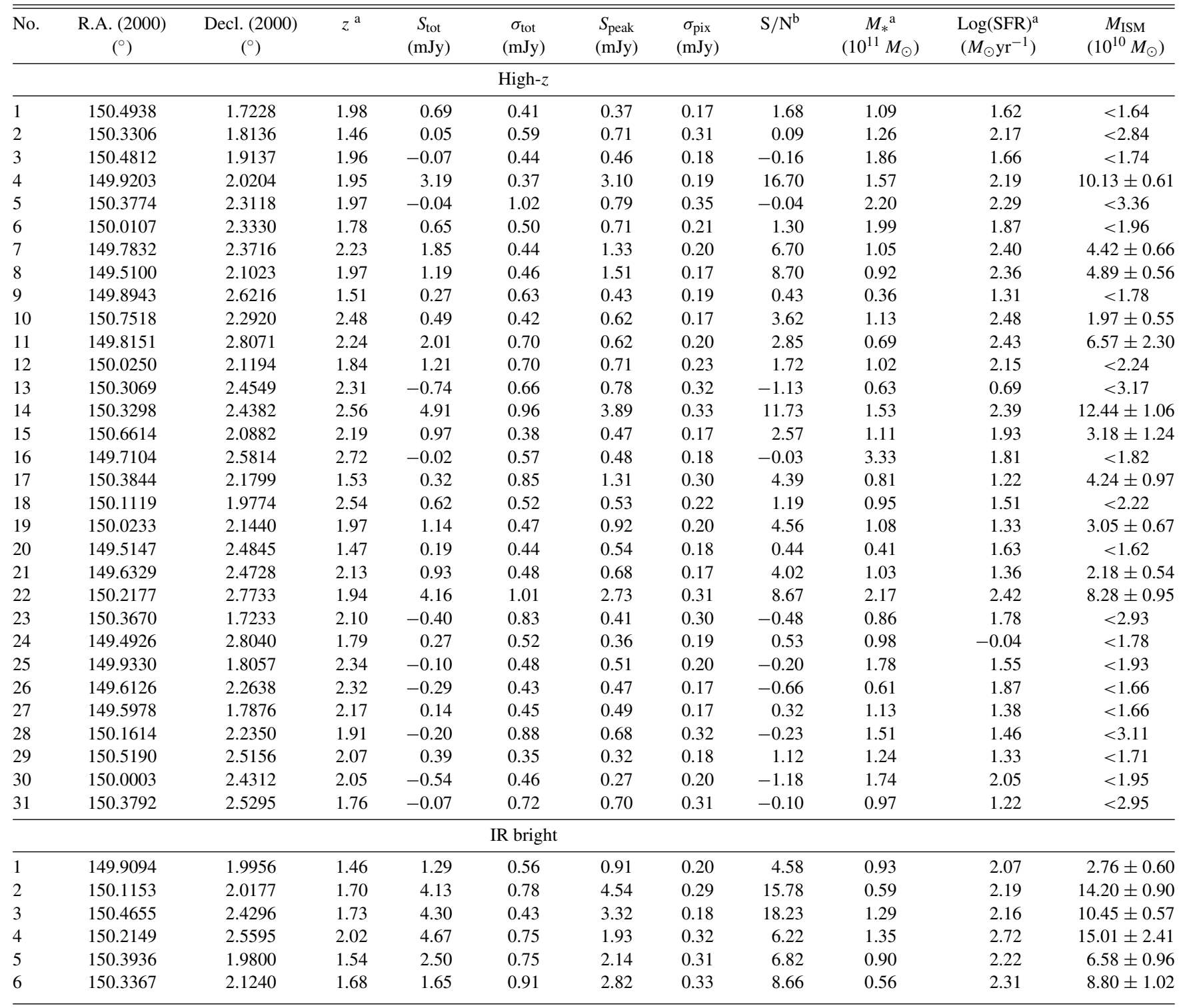

Notes.

${ }^{a}$ The photometric redshifts and stellar masses of the galaxies are from Ilbert et al. (2013) and the accuracy of those quantities is discussed in detail there. The SFRs are derived from the rest-frame UV continuum and the IR using Herschel PACS and SPIRE data, as detailed in Scoville et al. (2013). All of the galaxies have greater than $10 \sigma$ photometry measurements so the uncertainties in $M_{*}$ and SFR associated with their measurements are always less than $10 \%$. As discussed in Ilbert et al. (2013), the uncertainties in models used to derive the $M_{*}$ and SFR from the photometry are generally much larger but generally less than a factor two.

${ }^{b} \mathrm{~S} / \mathrm{N}_{\text {tot }}$ and $\mathrm{S} / \mathrm{N}_{\text {peak }}$ calculated separately from Equation (14) and the column $\mathrm{S} / \mathrm{N}$ lists the larger in absolute magnitude of those two S/Ns. Note that we let the $\mathrm{S} / \mathrm{N}$ be negative in cases where the flux estimate is negative so that several sigma negative flux values do not end up with a positive $\mathrm{S} / \mathrm{N}$ above the detection thresholds.

with accurate photometric redshifts, such as those in COSMOS.

Although the dust continuum can yield accurate and fast measurements of ISM masses, it is important to remember that the spectral line measurements do provide vital information such as dynamical masses, excitation, and abundance information-clearly these are not available from a single continuum measurement.

We thank the referee for a number of useful suggestions. We also thank Zara Scoville for proofreading the manuscript and Andreas Schruba for helpful comments. The Aspen Center for
Physics is acknowledged for hospitality during the initial writing of this paper; NSF grant 1066293 is also acknowledged. K.S.S. and K.S. are supported by the National Radio Astronomy Observatory, which is a facility of the National Science Foundation operated under cooperative agreement by Associated Universities, Inc. This paper makes use of the following ALMA data: ADS/JAO.ALMA\# 2011.0.00097.S. ALMA is a partnership of ESO (representing its member states), NSF (USA), and NINS (Japan), together with NRC (Canada) and NSC and ASIAA (Taiwan), in cooperation with the Republic of Chile. The Joint ALMA Observatory is operated by ESO, AUI/NRAO, and NAOJ. 


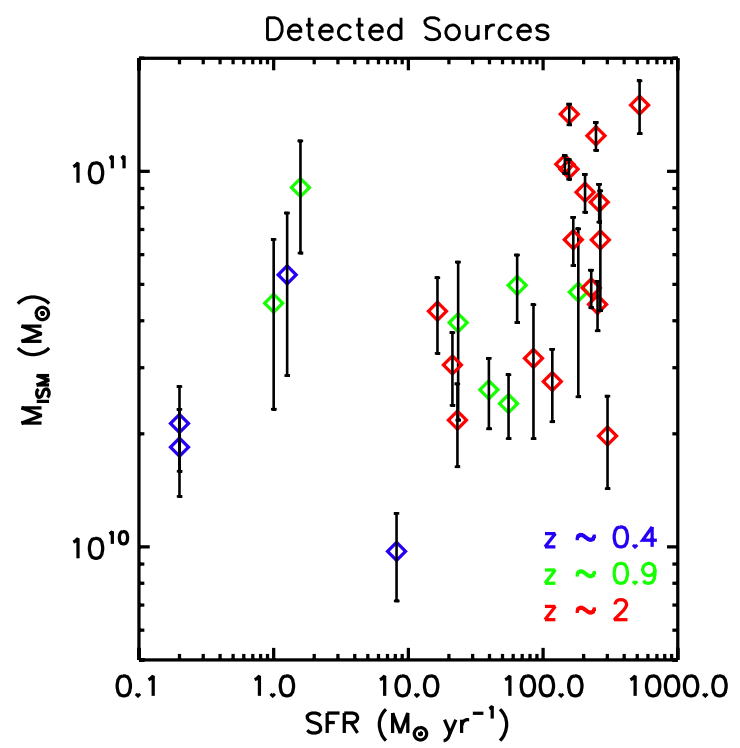

Figure 9. Inferred ISM masses are shown for the detected galaxies along with their SFRs derived from the COSMOS rest-frame UV continuum combined with the Herschel PACS and SPIRE fluxes. The redshift bin of each object is indicated by color coding. (For the two lowest SFR sources, their nominal SFRs are actually 10 times lower than shown but they were plotted at SFR = $0.2 M_{\odot} \mathrm{yr}^{-1}$ to maintain a reasonable scale for the other points. Uncertainties in the fluxes and $\mathrm{S} / \mathrm{Ns}$ are give in Tables 5-7.)

(A color version of this figure is available in the online journal.)

\section{APPENDIX}

\section{INDIVIDUAL GALAXIES AND THEIR FLUXES}

In Tables 5-7, we list the individual flux measurements and galaxy properties are summarized for all 107 galaxies in our survey. The objects are taken from the COSMOS survey field (Scoville et al. 2007) and the galaxy properties are from the latest photometric redshift catalog (Ilbert et al. 2013). This catalog has very high accuracy photometric redshifts based on very deep 34 band photometry, including near-IR photometry from the Ultra-Vista survey. See Ilbert et al. (2013) for accuracy of the redshifts and the derived galaxy stellar masses. The SFRs in Column 9 are from Scoville et al. (2013); they were derived from both the UV continuum and the Herschel PACS and SPIRE data.

Columns 5 and 6 in each table list integrated and peak flux measurements for apertures with $3^{\prime \prime}$ (Low- $z$ and Mid- $z$ ) and $2^{\prime \prime}($ High $-z)$ diameters centered on the galaxy position. The aperture sizes are intended to include most of a galactic disk $(\sim 10 \mathrm{kpc})$. The noise estimate in both cases was from the measured dispersion in the integrated and peak flux measurements obtained for 100 displaced off-center apertures of the same size in each individual image. The $\mathrm{S} / \mathrm{N}$ given in Column 7 is the better of those obtained from the integrated or peak flux measurement; it is the ratio of the signal in Columns 5 and 6 to the measured noise for Columns 5 and 6 . In the last column, limits are given at $2 \sigma$ and $3.6 \sigma$ in the inferred mass, depending on whether the better $\mathrm{S} / \mathrm{N}$ was obtained for the integrated or peak flux measurement. The detection thresholds of 2 and $3.6 \sigma$ are chosen such that the chance of a spurious "noise" detection across the entirety of each sample is less than $\sim 10 \%$ (based on the measured noise in each image and for the peak flux measurement, based on the number of pixels).

For the detected objects, Figure 9 shows their derived ISM masses based on the dust continuum and their SFRs.

\section{REFERENCES}

Aravena, M., Murphy, E. J., Aguirre, J. E., et al. 2013, MNRAS, 433, 498 Auld, R., Bianchi, S., Smith, M. W. L., et al. 2013, MNRAS, 428, 1880 Baker, A. J., Tacconi, L. J., Genzel, R., Lehnert, M. D., \& Lutz, D. 2004, ApJ, 604,125

Bothwell, M. S., Smail, I., Chapman, S. C., et al. 2013, MNRAS, 429, 3047 Boulanger, F., Abergel, A., Bernard, J.-P., et al. 1996, A\&A, 312, 256 Bussmann, R. S., Pérez-Fournon, I., Amber, S., et al. 2013, ApJ, 779, 25 Carilli, C., \& Walter, F. 2013, ARA\&A, 51, 105

Chapin, E. L., Pope, A., Scott, D., et al. 2009, MNRAS, 398, 1793

Clements, D. L., Dunne, L., \& Eales, S. 2010, MNRAS, 403, 274

Coppin, K. E. K., Smail, I., Alexander, D. M., et al. 2009, MNRAS, 395, 1905

Dale, D. A., Aniano, G., Engelbracht, C. W., et al. 2012, ApJ, 745, 95

Dale, D. A., Bendo, G. J., Engelbracht, C. W., et al. 2005, ApJ, 633, 857

Downes, D., Solomon, P. M., \& Radford, S. J. E. 1993, ApJL, 414, L13

Draine, B. T. 2011, Physics of the Interstellar and Intergalactic Medium (Princeton, NJ: Princeton Univ. Press)

Draine, B. T., Dale, D. A., Bendo, G., et al. 2007, ApJ, 663, 866

Draine, B. T., \& Li, A. 2007, ApJ, 657, 810

Dunne, L., \& Eales, S. A. 2001, MNRAS, 327, 697

Dunne, L., Gomez, H. L., da Cunha, E., et al. 2011, MNRAS, 417, 1510

Eales, S., Smith, M. W. L., Auld, R., et al. 2012, ApJ, 761, 168

Erb, D. K., Shapley, A. E., Pettini, M., et al. 2006, ApJ, 644, 813

Fu, H., Cooray, A., Feruglio, C., et al. 2013, Natur, 498, 338

Galametz, M., Madden, S. C., Galliano, F., et al. 2011, A\&A, 532, A56

Greve, T. R., Ivison, R. J., \& Papadopoulos, P. P. 2003, ApJ, 599, 839

Harris, A. I., Baker, A. J., Frayer, D. T., et al. 2012, ApJ, 752, 152

Harris, A. I., Baker, A. J., Zonak, S. G., et al. 2010, ApJ, 723, 1139

Hopkins, A. M., \& Beacom, J. F. 2006, ApJ, 651, 142

Ilbert, O., McCracken, H. J., Le Fèvre, O., et al. 2013, A\&A, 556, A55

Ivison, R. J., Papadopoulos, P. P., Smail, I., et al. 2011, MNRAS, 412, 1913

Ivison, R. J., Swinbank, A. M., Smail, I., et al. 2013, ApJ, 772, 137

Lestrade, J.-F., Carilli, C. L., Thanjavur, K., et al. 2011, ApJL, 739, L30

Magdis, G. E., Daddi, E., Béthermin, M., et al. 2013, ApJ, 760, 6

Planck Collaboration. 2011a, A\&A, 536, A21

Planck Collaboration. 2011b, A\&A, 536, A25

Riechers, D. A., Cooray, A., Omont, A., et al. 2011a, ApJL, 733, L12

Riechers, D. A., Hodge, J., Walter, F., Carilli, C. L., \& Bertoldi, F. 2011b, ApJL, 739, L31

Scoville, N., Arnouts, S., Aussel, H., et al. 2013, ApJS, 206, 3

Scoville, N., Aussel, H., Brusa, M., et al. 2007, ApJS, 172, 1

Scoville, N. Z. 2012, arXiv: 1210.6990

Scoville, N. Z., Yun, M. S., Sanders, D. B., Clemens, D. P., \& Waller, W. H. 1987, ApJS, 63, 821

Solomon, P. M., Rivolo, A. R., Barrett, J., \& Yahil, A. 1987, ApJ, 319, 730

Solomon, P. M., \& Vanden Bout, P. A. 2005, ARA\&A, 43, 677

Tacconi, L. J., Genzel, R., Nevi, R., et al. 2010, Natur, 463, 781

Tacconi, L. J., Neri, R., Genzel, R., et al. 2013, ApJ, 768, 74

Thomson, A. P., Ivison, R. J., Smail, I., et al. 2012, MNRAS, 425, 2203 